Mon. Not. R. Astron. Soc. 000,1-?? (2018) Printed 18 March $2021 \quad$ (MN LATEX style file v2.2)

\title{
NGC 6611 601: A hot pre-main sequence spectroscopic binary containing a centrifugal magnetosphere host star
}

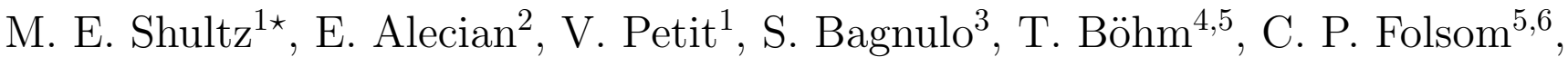 \\ G. A. Wade ${ }^{6}$, and the MiMeS Collaboration \\ ${ }^{1}$ Department of Physics and Astronomy, University of Delaware, 217 Sharp Lab, Newark, Delaware, 19716, USA \\ ${ }^{2}$ Université Grenoble Alpes, IPAG, F-38000 Grenoble, France \\ ${ }^{4}$ Université de Toulouse; UPS-OMP; IRAP; Toulouse, France \\ ${ }^{5}$ CNRS; IRAP; 14, avenue Edouard Belin, 31400 Toulouse, France \\ ${ }^{6}$ Department of Physics and Space Science, Royal Military College of Canada, Kingston, Ontario K7K 7B4, Canada
}

\begin{abstract}
W 601 (NGC 6611601) is one of the handful of known magnetic Herbig Ae/Be stars. We report the analysis of a large dataset of high-resolution spectropolarimetry. The star is a previously unreported spectroscopic binary, consisting of 2 B2 stars with a mass ratio of 1.8 , masses of $12 \mathrm{M}_{\odot}$ and $6.2 \mathrm{M}_{\odot}$, in an eccentric 110-day orbit. The magnetic field belongs to the secondary, $\mathrm{W} 601 \mathrm{~B}$. The $\mathrm{H} \alpha$ emission is consistent with an origin in W601 B's centrifugal magnetosphere; the star is therefore not a classical Herbig Be star in the sense that its emission is not formed in an accretion disk. However, the low value of $\log g=3.8$ determined via spectroscopic analysis, and the star's membership in the young NGC 6611 cluster, are most consistent with it being on the pre-main sequence. The rotational period inferred from the variability of the $\mathrm{H} \alpha$ line and the longitudinal magnetic field $\left\langle B_{z}\right\rangle$ is $1.13 \mathrm{~d}$. Modelling of Stokes $V$ and $\left\langle B_{z}\right\rangle$ indicates a surface dipolar magnetic field $B_{\mathrm{d}}$ between 6 and $11 \mathrm{kG}$. With its strong emission, rapid rotation, and strong surface magnetic field, W $601 \mathrm{~B}$ is likely a precursor to $\mathrm{H} \alpha$-bright magnetic B-type stars such as $\sigma$ Ori E. By contrast, the primary is an apparently non-magnetic $\left(B_{\mathrm{d}}<300 \mathrm{G}\right)$ pre-main sequence early Btype star. In accordance with expectations from magnetic braking, the non-magnetic primary is apparently more rapidly rotating than the magnetic star.
\end{abstract}

Key words: stars: individual: NGC 6611601 - stars: early-type - stars: magnetic field - stars: massive - stars: rotation

\section{INTRODUCTION}

Approximately $10 \%$ of main sequence and pre-main sequence stars with radiative envelopes host strong $(\sim \mathrm{kG}$ scale), organized (predominantly dipolar) magnetic fields (Grunhut et al. 2017; Sikora et al. 2019a). Since radiative envelopes cannot support a contemporaneous convective dynamo, it is believed that the magnetic fields of early type stars are fossils - remnants from a previous stage in the stars' evolution (e.g. Braithwaite \& Spruit 2004; Neiner et al. 2015). Consistent with the fossil field hypothesis is that, unlike dynamo magnetic fields, hot star magnetic fields are apparently stable over a time-span of at least decades (e.g. Shultz et al. 2018b), show no general correlation between magnetic field strength and rotational properties, and exhibit a decline in surface magnetic field strength

* E-mail: mshultz@udel.edu over evolutionary timescales consistent with conservation or slow decay of magnetic flux (e.g. Landstreet et al. 2007, 2008; Sikora et al. 2019b; Shultz et al. 2019d).

The origin of fossil magnetic fields remains obscure. One scenario is that they arise due to short-lived dynamos generated during binary mergers (e.g. Schneider et al. 2019), however this is difficult to reconcile with the orbital properties of known close magnetic binary systems such as the doubly-magnetic system $\epsilon$ Lupi (Shultz et al. 2015b), the tidally locked system HD 98088 (Folsom et al. 2013), the very close binary HD 156324 (Shultz et al. 2018a), or the 'identical twins' of HD 62658 (Shultz et al. 2019e). A competing scenario is that fossil fields may be remnants of convective dynamos operating on the pre-main sequence (PMS) (e.g. Mestel 1999), in which case the $10 \%$ incidence ratio might perhaps be explained by a PMS dynamo bistability mechanism similar to that seen in fully convective stars (as discussed by Shultz et al. 2019e) combined with rapid rota- 
tionally or convectively driven decay of magnetic fields failing to reach critical surface strength (Aurière et al. 2007; Jermyn \& Cantiello 2020).

This makes examination of magnetic hot stars on the PMS a key arena for determining the origin of fossil magnetic fields. Only eight PMS early-type stars with magnetic field detections confirmed with highresolution spectropolarimetry are known (Wade et al. 2005; Petit et al. 2008; Catala et al. 2007; Alecian et al. 2008a|b; Hubrig et al. 2009; Alecian et al. 2013a; Hubrig et al. 2013, 2015; Järvinen et al. 2015). Alecian et al. (2013a,b) showed that fossil magnetic fields have the same incidence amongst Herbig Ae/Be stars as amongst the MS population, and tend to be more slowly rotating than non-magnetic Herbig stars, which is consistent with the magnetic stars being subject to magnetic braking. A spectropolarimetric survey of intermediate mass T Tauri stars by Villebrun et al. (2019) demonstrated that magnetic fields are ubiquitous amongst stars with convective envelopes, but that the incidence declines to around $10 \%$ immediately upon crossing the boundary on the Hertzsprung-Russell diagram between convective and radiative envelopes.

Since only a few magnetic PMS hot stars are known, a full magnetic and rotational characterization of the individual members of this population is an important step in understanding their properties. In this paper, we examine the PMS B2 star NGC6611601 (also known as NGC 6611-019, ALS 9522, BD - $13^{\circ}$ 4937, and hereafter referred to as W601). This star was classified as a Herbig $\mathrm{Ae} / \mathrm{Be}$ star on the basis of its 'P-Cygni like' $\mathrm{H} \alpha$ emisison (Martavan et al. 2008) and its mid-infrared excess (Kumar et al. 2004). Its magnetic field was detected by Alecian et al. (2008b), who also noted its strong, variable $\mathrm{He}$ lines, suggesting that it may be a He-strong star. W 601 is an X-ray source (Nazé et al. 2014), and a radio synchrotron source (Kurapati et al. 2017), both likely indicative that the star hosts a detectable magnetosphere; the other possible explanation for enhanced X-rays and non-thermal radio emission - colliding winds - seems unlikely due to the relatively weak winds of B-type stars, and unneccessary due to the presence of a magnetic field.

The observations - a large ESPaDOnS dataset - are described in $\S 2$. In $\S 3$ we show the evidence that $\mathrm{W} 601$ is a spectroscopic binary, and constrain the orbital properties of the system. Stellar parameters are revisted in the light of binarity in $\S 4$ The magnetic analysis is presented in $\S 5$. where it is determined that the magnetic field belongs to the secondary. Spectroscopic variability is examined in $\S[$ and in $\S[$ the rotational period is determined from the magnetic and spectroscopic variations. The magnetic properties of the stars are derived in $\S 8$. The magnetospheric properties of the system, and its possible future magnetic and rotational evolution, are discussed in $\S 9$, along with implications for the origins of fossil magnetic fields, their accompanying chemical abundance anomalies, and the status of the system as a Herbig star. Conclusions are summarized in $\S 10$

\section{OBSERVATIONS}

The dataset consists of 27 spectropolarimetric ESPaDOnS sequences obtained at the $3.6 \mathrm{~m}$ Canada-France-Hawaii
Telescope (CFHT) from several observing programs (program codes 06BF15, 07AF06, 08AF16, 08AC13, 08BP14, $12 \mathrm{AP} 13$, and 13BC09), and in the context of the Magnetism in Massive Stars (MiMeS) Large Program. ESPaDOnS is a fibre-fed echelle spectropolarimeter with a spectral resolution $\lambda / \Delta \lambda \sim 65,000$ at $500 \mathrm{~nm}$, and a wavelength coverage between about $370 \mathrm{~nm}$ and $1050 \mathrm{~nm}$. Each observation consists of 4 polarized sub-exposures, which are combined to yield a circular polarization (Stokes $V$ ) spectrum and two diagnostic null $N$ spectra, with which anomalies in instrument behaviour can be detected. The reduction and analysis of ESPaDOnS data was described in detail by Wade et al. (2016). The data were reduced with the LibreESPRIT pipeline (Donati et al. 1997).

The observation log is given in Table 1. The mean peak signal-to-noise $(S / N)$ per spectral pixel is about 200 .

Four of these observations were already reported by Alecian et al. (2008b) (one on 10/08/2006, two on $06 / 03 / 2007$, and one on $09 / 03 / 07)$. The remaining observation are presented here for the first time.

\section{BINARITY}

Magnetic Bp stars exhibit several forms of line profile variation. The first, and most common in this class, are due to inhomogeneous surface chemical abundance patches, almost invariably exhibiting different patterns of variation for different elements (e.g. Kochukhov et al. 2015; Rusomarov et al. 2015, 2016; Silvester et al. 2017; Rusomarov et al. 2018; Kochukhov et al. 2019). Many Bp stars also exhibit line profile variability due to pulsation or binarity. As is shown below, the line profile variations of W601 reported by Alecian et al. (2008b), and interpted there as the signature of He spots, are in fact primarily due to binarity.

Close examination of the line profile variability shows that lines of different elements exhibit essentially the same pattern of variation (Fig. 1), with absorption excesses appearing in the same part of the line regardless of element. Since it is not expected that different elements will display the same abundance distributions, the similarity of the line profile variability between lines from different elements suggests that the source of the variation may instead be either binarity or pulsation.

To test the binarity hypothesis, two-component models were fit to the strong He I $667.8 \mathrm{~nm}$ line using the paramaterized line profile fitting routine described by Grunhut et al. (2017). This process yielded consistent fits for a broad-lined component $\left(v \sin i=173 \pm 14 \mathrm{~km} \mathrm{~s}^{-1}\right)$ and a narrow-lined component $\left(v \sin i=105 \pm 3 \mathrm{~km} \mathrm{~s}^{-1}\right)$, where the uncertainties are determined from the standard deviation of fits across the dataset. The radial velocities (RVs) of the two components obtained in the course of profile fitting anticorrelate with one another (Fig. 2, top). Since the broad-lined component has the smaller RV amplitude, we designate it as the primary, W601 A, and the narrow-lined component as W $601 \mathrm{~B}$. W $601 \mathrm{~A}$ has an RV semi-amplitude of about $20 \mathrm{~km} \mathrm{~s}^{-1}$ and $\mathrm{W} 601 \mathrm{~B}$ has a semi-amplitude of about 30 $\mathrm{km} \mathrm{s}^{-1}$. There is no detectable RV variation on a time-scale of one to two days, however there is significant variation over a time-scale of weeks. This is in contrast to EW variations 
Table 1. Observation log, RV measurements, and $\left\langle B_{z}\right\rangle$ measurements. $S / N$ indicates the maximum signal-to-noise per spectral pixel in the ESPaDOnS spectrum. Average RV uncertainties are $1.8 \mathrm{~km} \mathrm{~s}^{-1}$ for W $601 \mathrm{~A}$ and $1.5 \mathrm{~km} \mathrm{~s}^{-1}$ for W $601 \mathrm{~B}$. DF refers to the magnetic field detection flag (DD: Definite Detection; MD: Marginal Detection; ND: Non-Detection).

\begin{tabular}{|c|c|c|c|c|c|c|c|c|}
\hline Date & $\begin{array}{r}\text { HJD- } \\
-2453000\end{array}$ & $S / N$ & $\begin{array}{r}\mathrm{RV}_{\mathrm{A}} \\
(\mathrm{km} / \mathrm{s})\end{array}$ & $\begin{array}{r}\mathrm{RV}_{\mathrm{B}} \\
(\mathrm{km} / \mathrm{s})\end{array}$ & $\begin{array}{r}\left\langle B_{z}\right\rangle \\
(\mathrm{G})\end{array}$ & $\mathrm{DF}_{V}$ & $\begin{array}{r}\left\langle N_{z}\right\rangle \\
(\mathrm{G})\end{array}$ & $\mathrm{DF}_{N}$ \\
\hline $10 / 08 / 2006$ & 957.76004 & 220 & 5 & 20 & $184 \pm 286$ & DD & $-139 \pm 286$ & ND \\
\hline $02 / 03 / 2007$ & 1162.15478 & 103 & -7 & 38 & $-709 \pm 1015$ & ND & $-1067 \pm 1015$ & ND \\
\hline $03 / 03 / 2007$ & 1163.12053 & 192 & 0 & 31 & $408 \pm 348$ & ND & $-644 \pm 348$ & ND \\
\hline $04 / 03 / 2007$ & 1164.12133 & 226 & -2 & 34 & $-857 \pm 286$ & ND & $-391 \pm 286$ & ND \\
\hline $05 / 03 / 2007$ & 1165.13840 & 254 & -6 & 41 & $-805 \pm 247$ & ND & $-450 \pm 247$ & ND \\
\hline $06 / 03 / 2007$ & 1166.10187 & 179 & -6 & 45 & $-511 \pm 343$ & ND & $116 \pm 343$ & ND \\
\hline $06 / 03 / 2007$ & 1166.14879 & 153 & -7 & 47 & $-458 \pm 617$ & ND & $213 \pm 617$ & ND \\
\hline $07 / 03 / 2007$ & 1167.11236 & 182 & -9 & 49 & $-117 \pm 362$ & MD & $59 \pm 362$ & ND \\
\hline $08 / 03 / 2007$ & 1168.10114 & 194 & -6 & 45 & $1424 \pm 354$ & DD & $-508 \pm 354$ & ND \\
\hline $08 / 03 / 2007$ & 1168.14652 & 204 & -9 & 47 & $921 \pm 332$ & DD & $606 \pm 332$ & ND \\
\hline $09 / 03 / 2007$ & 1169.10634 & 190 & 0 & 38 & $2014 \pm 349$ & DD & $-813 \pm 348$ & ND \\
\hline $09 / 03 / 2007$ & 1169.15224 & 199 & -1 & 36 & $2036 \pm 370$ & DD & $-246 \pm 369$ & ND \\
\hline $30 / 06 / 2008$ & 1647.80106 & 203 & 35 & -30 & $2095 \pm 311$ & DD & $547 \pm 311$ & ND \\
\hline $30 / 06 / 2008$ & 1647.84933 & 191 & 35 & -30 & $2079 \pm 335$ & DD & $336 \pm 334$ & ND \\
\hline $30 / 06 / 2008$ & 1647.89851 & 185 & 33 & -28 & $1784 \pm 354$ & DD & $31 \pm 354$ & ND \\
\hline $30 / 06 / 2008$ & 1647.94664 & 175 & 31 & -25 & $1797 \pm 393$ & $\mathrm{DD}$ & $-589 \pm 392$ & ND \\
\hline $30 / 06 / 2008$ & 1647.99560 & 179 & 28 & -19 & $443 \pm 359$ & MD & $-283 \pm 359$ & ND \\
\hline $30 / 06 / 2008$ & 1648.04373 & 179 & 29 & -19 & $1178 \pm 382$ & $\mathrm{DD}$ & $-33 \pm 382$ & ND \\
\hline $01 / 07 / 2008$ & 1648.80655 & 209 & 31 & -30 & $1446 \pm 311$ & DD & $200 \pm 310$ & ND \\
\hline $01 / 07 / 2008$ & 1648.85518 & 205 & 31 & -26 & $1808 \pm 325$ & DD & $17 \pm 324$ & ND \\
\hline $01 / 07 / 2008$ & 1648.90492 & 202 & 32 & -28 & $2509 \pm 327$ & DD & $-499 \pm 326$ & $\mathrm{ND}$ \\
\hline $01 / 07 / 2008$ & 1648.95328 & 205 & 31 & -25 & $2359 \pm 320$ & DD & $-212 \pm 320$ & ND \\
\hline $01 / 07 / 2008$ & 1649.00232 & 219 & 33 & -28 & $2053 \pm 291$ & DD & $6 \pm 290$ & ND \\
\hline $01 / 07 / 2008$ & 1649.05057 & 213 & 31 & -25 & $1748 \pm 299$ & DD & $101 \pm 299$ & ND \\
\hline $29 / 07 / 2008$ & 1676.89812 & 167 & 9 & 15 & $370 \pm 412$ & ND & $-430 \pm 412$ & ND \\
\hline $21 / 06 / 2012$ & 3099.93880 & 272 & 24 & -8 & $2088 \pm 308$ & DD & $-170 \pm 307$ & ND \\
\hline $13 / 08 / 2013$ & 3517.80010 & 264 & 34 & -27 & $1256 \pm 329$ & DD & $480 \pm 329$ & ND \\
\hline
\end{tabular}

tracing rotational variability, which occur on a much shorter timescale (see $\S$ 6).

The RVs and $v \sin i$ values were then used to disentangle the line profiles of various spectral lines using the iterative method described by González \& Levato (2006), as shown for the He I $667.8 \mathrm{~nm}$, Si III $455.3 \mathrm{~nm}$, and Si II $634.7 \mathrm{~nm}$ lines in Fig. 1. As can be seen in Fig. 11 the broad-lined component accounts for the majority of line absorption: $57 \%$ of the absorption in He I $667.8 \mathrm{~nm}, 74 \%$ in Si III $455.3 \mathrm{~nm}$, and $63 \%$ in Si II $634.7 \mathrm{~nm}$.

The anticorrelation of the RVs, and the fact that the larger RV amplitude is seen in the component with the smaller contribution to the line absorption, are all consistent with W 601 being a double-lined spectroscopic binary (SB2).

Period analyses of the RVs were performed using both the standard Fourier analysis package PERIOD04 Lenz \& Breger 2005) and using the IDL routine PERIODOGRAM.PRd (Horne \& Baliunas 1986). Period uncertainties were determined analytically (Bloomfield 1976). Analysis of the full RV dataset yielded ambiguous results, with numerous peaks of similar amplitude between about 30 and 200 days. Since there is only minimal variation on timescales of a few days (Fig. 2, left panel), we analyzed mean weekly RVs in order to avoid biasing the periodogram

1 Available at https://www.univie.ac.at/tops/Period04/ 2 Available at https://hesperia.gsfc.nasa.gov/ssw/gen/idl/util/periooddgrapito semi-amplitudes $K$, the systemic velocities results by the few highly sampled epochs. This strategy yielded a period of 109.9(2) d from both the A and B RVs (see Fig. 3 where periodograms from PERIODOGRAM.PRO are shown). PERIOD04 instead yielded a period of $104 \mathrm{~d}$, however, the longer period obtained using PERIODOGRAM.PRO provides a better phasing of the data (Fig. 2, right). The longer period furthermore yields a higher $S / N$ as evaluated using PERIOD04 itself (26 as compared to 5.5). We therefore adopt the period from PERIODOGRAM.PRO as the most likely orbital period, although we note that there is enough ambiguity in the period that it should certainly be tested by further observation.

To obtain an orbital solution, we optimized synthetic RV curves against the mean weekly RVs using a Markov Chain Monte Carlo algorithm (as described by Shultz et al. 2018a, 2019a). The algorithm achieved rapid convergence, providing additional confidence in the period. The model fits to the RVs are shown in the right panel of Fig. 2, and the fit parameters are given in Table 2 The orbit is mildly eccentric $(e=0.24 \pm 0.01)$, with a mass ratio $M_{\mathrm{A}} / M_{\mathrm{B}}=1.79 \pm 0.04$. The $\chi^{2}$ of the fit shown in Fig. 2 is 3.6. MCMC fitting of the $104 \mathrm{~d}$ period yielded a $\chi^{2}$ of 15.4 , with a noticeably worse fit. The periodogram also has a peak at $186 \mathrm{~d}$, nearly the same strength as the $109 \mathrm{~d}$ period; fitting with this period yielded a $\chi^{2}$ of 6.7 , somewhat higher than the $109 \mathrm{~d}$ period. This indicates that the $109 \mathrm{~d}$ period is the most likely to be correct. Notably, while the 3 fits yield different eccentricities 

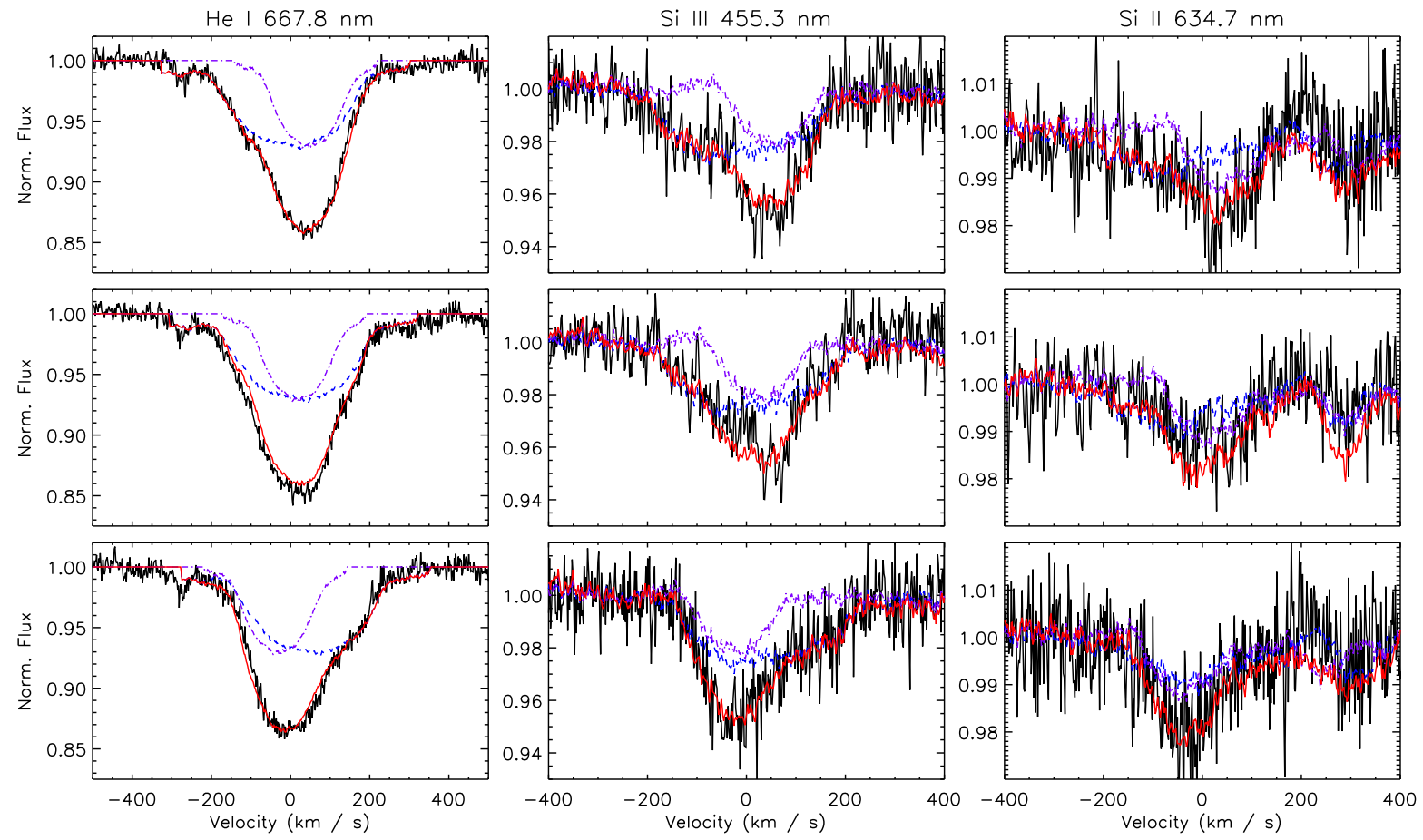

Figure 1. Observed line profiles (black lines) and disentangled line profiles (dashed blue: primary; dot-dashed purple: secondary; solid red: cumlative) for 3 isolated spectral lines. Each row shows the same spectrum. The top, middle, and bottom rows respectively show spectra with the secondary at maximum positive radial velocity, the minimum difference in radial velocity between primary and secondary, and with the secondary at maximum negative radial velocity. Line profile variations in all lines can be reproduced assuming a two-star model with variable RVs.
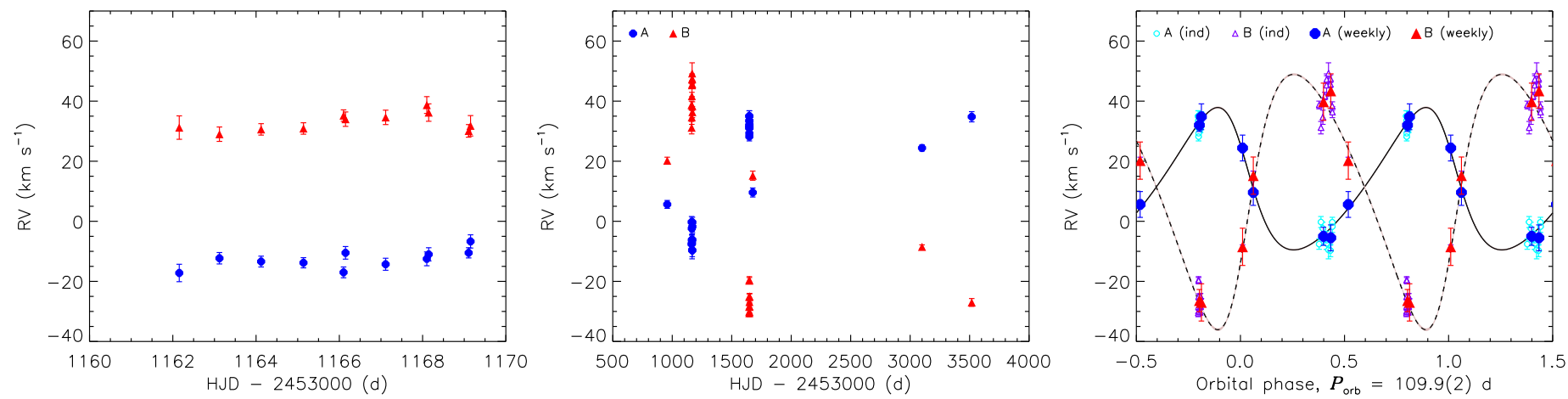

Figure 2. Radial velocities for the primary (blue circles) and secondary (red triangles) as a function of time (left, middle) and phased with the orbital period (right). The left panel zooms in on the epoch with the densest time sampling. In the right panel, curves and shaded regions indicate orbital models and uncertainties.

$v_{0}$ and the mass ratios $M_{\mathrm{A}} / M_{\mathrm{B}}=K_{\mathrm{B}} / K_{\mathrm{A}}$ are essentially identical in all three cases, indicating that these parameters are likely robust against future revision of the orbital period.

\section{STELLAR PARAMETERS}

Stellar parameters for W 601 have previously been determined by de Winter et al. (1997) $\left(\log L / L_{\odot}=4.28, T_{\text {eff }}=\right.$ $23.5 \mathrm{kK})$ and Dufton et al. (2006) $\left(\log L / L_{\odot}=3.96 \pm 0.11\right.$, $T_{\text {eff }}=22.4 \pm 2.7 \mathrm{kK}$, and $\left.\log g=3.85\right)$, where the different luminosities arise from different assumptions regarding distance and extinction. Since these parameters were determined under the assumption of a single star, they need to be revisited.

\subsection{Effective Temperature}

As a first pass to estimate the $T_{\text {eff }}$ of the two components, we used EW ratios of Si II $634.7 \mathrm{~nm}$ and Si III $455.3 \mathrm{~nm}$ obtained from disentangled line profiles (Fig. 1). While surface abundances are by definition affected by the chemical spots expected for a magnetic chemically peculiar star, line strength ratios for different ions of the same element are not 


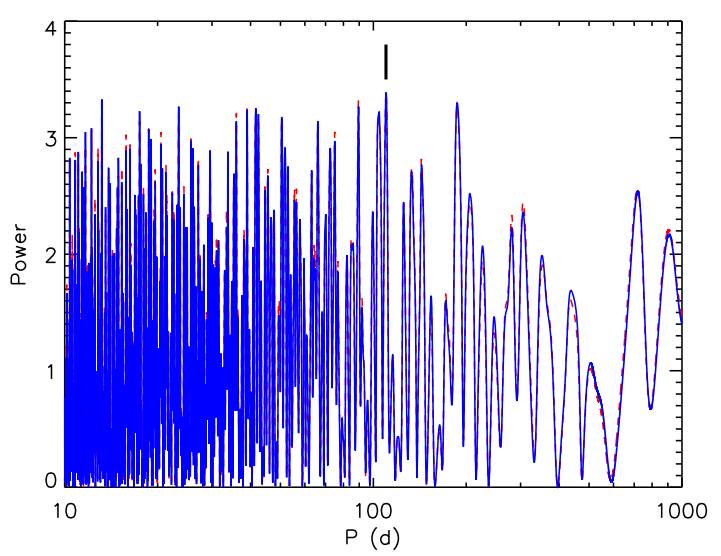

Figure 3. Periodograms for the primary (solid blue) and secondary (dashed red) mean weekly RVs. The thick black line indicates the maximum power period. Note that there are several peaks of nearly equivalent significance; these are discussed further in the text.

Table 2. Orbital parameters: orbital period $P_{\text {orb }}$; epoch of periastron $T_{0}$; eccentricity $e$; argument of periastron $\omega$; central velocity $v_{0}$; semi-amplitudes $K_{\mathrm{A}}$ and $K_{\mathrm{B}} ;$ mass ratio $M_{\mathrm{A}} / M_{\mathrm{B}}$; projected total mass $M \sin ^{3} i$; projected component masses $M_{\mathrm{A}} \sin ^{3} i$ and $M_{\mathrm{B}} \sin ^{3} i$; and projected semi-major axis $a \sin i$.

\begin{tabular}{lrr}
\hline \hline Parameter & Value & Uncertainty \\
\hline$P_{\text {orb }}(\mathrm{d})$ & 109.9 & 0.2 \\
$T_{0}(\mathrm{HJD})$ & 2453900.8 & 0.2 \\
$e$ & 0.234 & 0.008 \\
$\omega\left(^{\circ}\right)$ & 64.6 & 0.9 \\
$v_{0}(\mathrm{~km} / \mathrm{s})$ & 11.4 & 0.1 \\
$K_{\mathrm{A}}(\mathrm{km} / \mathrm{s})$ & 21.5 & 0.2 \\
$K_{\mathrm{B}}(\mathrm{km} / \mathrm{s})$ & 41.8 & 0.4 \\
$M_{\mathrm{A}} / M_{\mathrm{B}}$ & 1.94 & 0.03 \\
$M \sin ^{3} i\left(\mathrm{M}_{\odot}\right)$ & 2.64 & 0.06 \\
$M_{\mathrm{A}} \sin ^{3} i\left(\mathrm{M}_{\odot}\right)$ & 1.75 & 0.04 \\
$M_{\mathrm{B}} \sin ^{3} i\left(\mathrm{M}_{\odot}\right)$ & 0.90 & 0.02 \\
$a \sin i(\mathrm{AU})$ & 0.621 & 0.005 \\
\hline \hline
\end{tabular}

affected by this (since it is the abundance, but not the $T_{\text {eff }}$, that varies across the stellar surface; e.g. Shultz et al. 2015a, $2019 \mathrm{~b}$ ). Comparison of the weighted mean EW ratios calculated from all spectra to theoretical values obtained from the NLTE TLUSTY BSTAR2006 library of synthetic spectra (Lanz \& Hubenv 2007), using $3.5<\log g<4.25$, yields $T_{\text {eff A }}=22.5 \pm 1.5 \mathrm{kK}$ and $T_{\text {eff B }}=20.5 \pm 1.5 \mathrm{kK}$. While the $T_{\text {eff }}$ of the two components overlaps within uncertainty, $\mathrm{B}$ is definitely cooler than $\mathrm{A}$, since the contribution of $\mathrm{A}$ is higher in Si III as compared to Si II.

A second analysis is demonstrated in Fig. 4, where we used a grid of synthetic spectra calculated from TLUSTY models, with $15<T_{\text {eff }}<25 \mathrm{kK}$ and $3.0<\log g<4.5$ for each star. The TLUSTY spectra were calculated with 2 $\mathrm{km} \mathrm{s}^{-1}$ of microturbulence and solar metallicity. The radius ratio $R_{\mathrm{A}} / R_{\mathrm{B}}$ was allowed to vary as a free parameter between 0.5 and 5 , where $R_{\mathrm{A}} / R_{\mathrm{B}}$ is a factor used to scale the contributions of the $\mathrm{A}$ and $\mathrm{B}$ components to the composite spectrum. $v \sin i_{\mathrm{A}}=173 \mathrm{~km} \mathrm{~s}^{-1}, v \sin i_{\mathrm{B}}=94 \mathrm{~km} \mathrm{~s}^{-1}$,
Table 3. Stellar parameters for the W601 components. ${ }^{a}$, Kuhn et al. (2019); ${ }^{b}$ : using the anomalous extinction law determined by Dufton et al. (2006); ${ }^{c}$ : Nieva (2013). Pre-Main Sequence (PMS) models: Haemmerlé et al. (2019); Main Sequence (MS) models: Ekström et al. (2012).

\begin{tabular}{|c|c|c|}
\hline Parameter & $\mathrm{A}$ & $\mathrm{B}$ \\
\hline$B$ (mag) & \multicolumn{2}{|c|}{11.11} \\
\hline$V$ (mag) & \multicolumn{2}{|c|}{10.78} \\
\hline$d(\mathrm{kpc})^{a}$ & \multicolumn{2}{|c|}{$1.74 \pm 0.13$} \\
\hline$E(B-V)$ & \multicolumn{2}{|c|}{$0.68 \pm 0.05$} \\
\hline$A_{\mathrm{V}}(\mathrm{mag})^{b}$ & \multicolumn{2}{|c|}{$2.38 \pm 0.3$} \\
\hline$M_{V}(\mathrm{mag})$ & $-3.0 \pm 0.4$ & $-1.7 \pm 0.3$ \\
\hline $\mathrm{BC}(\mathrm{mag})^{c}$ & $-2.6 \pm 0.1$ & $-1.6 \pm 0.2$ \\
\hline$M_{\mathrm{bol}}(\mathrm{mag})$ & $-5.7 \pm 0.4$ & $-3.3 \pm 0.3$ \\
\hline$T_{\text {eff }}(\mathrm{kK})$ & $22 \pm 1$ & $19 \pm 1$ \\
\hline $\log g(\mathrm{~cm} / \mathrm{s})$ & $3.9_{-0.1}^{+0.3}$ & $3.9_{-0.3}^{+0.5}$ \\
\hline $\log (L / L \odot)$ & $4.1 \pm 0.2$ & $3.2 \pm 0.1$ \\
\hline$R_{*}\left(\mathrm{R}_{\odot}\right)$ & $5.2 \pm 0.7$ & $4.3 \pm 0.9$ \\
\hline$M_{*}\left(\mathrm{M}_{\odot}\right)$ & $12 \pm 1$ & $6.2 \pm 0.4$ \\
\hline $\log (t / \mathrm{yr})(\mathrm{PMS})$ & \multicolumn{2}{|c|}{$5.6 \pm 0.2$} \\
\hline $\log (t / y r)(\mathrm{MS})$ & \multicolumn{2}{|c|}{$7.3 \pm 0.2$} \\
\hline$i_{\text {orb }}\left({ }^{\circ}\right)$ & \multicolumn{2}{|c|}{$31.5 \pm 0.9$} \\
\hline$a(\mathrm{AU})$ & \multicolumn{2}{|c|}{$1.18 \pm 0.02$} \\
\hline
\end{tabular}

$R V_{\mathrm{A}}=35 \mathrm{~km} \mathrm{~s}^{-1}$, and $\mathrm{RV}_{\mathrm{B}}=-23 \mathrm{~km} \mathrm{~s}^{-1}$ were fixed. The grid was compared to a mean spectrum created by combining the ESPaDOnS observations obtained in $03 / 2007$, since the RVs of the two components are basically constant during the 1 week of observations (see the left panel of Fig. 2. The standard deviation in RV is $3.5 \mathrm{~km} \mathrm{~s}^{-1}$ and $6 \mathrm{~km} \mathrm{~s}^{-1}$ for W $601 \mathrm{~A}$ and B, respectively, in both cases comparable to the uncertainty in $v \sin i$; co-addition of spectra therefore should not lead to significant additional line broadening)., and this is the epoch with the largest number of observations. The peak $S / N$ per $1.8 \mathrm{~km} \mathrm{~s}^{-1}$ spectral pixel of the mean 2007 spectrum is 670 . The analysis was performed on a selection of strong lines, shown in Fig. 4 with a focus on chemical species for which two ionization levels are present in the spectrum (O I and II; Si II and III; and Fe II and III). The results of this analysis are $T_{\text {eff,A }}=22 \pm 1 \mathrm{kK}$, $T_{\text {eff }, \mathrm{B}}=19 \pm 1 \mathrm{kK}, \log g=3.75 \pm 0.25$ for both stars, and $R_{\mathrm{A}} / R_{\mathrm{B}}=1.1 \pm 0.1$.

The results of the spectroscopic fit are consistent with those of the EW ratio analysis, but use a larger number of lines, and yield more precise results; we therefore adopt the spectroscopic fit results for the $T_{\text {eff }}$. The surface gravity is revisited below using Balmer lines, as these are more sensitive to $\log g$.

Alecian et al. (2008b) classified W 601 as a He-strong star because its He lines were much stronger than solar abundance models predict, as is usually the case for strongly magnetic stars in this $T_{\text {eff }}$ regime. As can be seen in Fig. 4. a binary spectroscopic model with solar abundances also results in He lines much weaker than observed. For this reason, He lines were not included in the $T_{\text {eff }}$ determination.

\subsection{Surface Gravity}

To refine the determination of $\log g$ we examined the $\mathrm{H} \beta$ and $\mathrm{H} \gamma$ lines. Since there is emission in $\mathrm{H} \alpha$, there will also be 

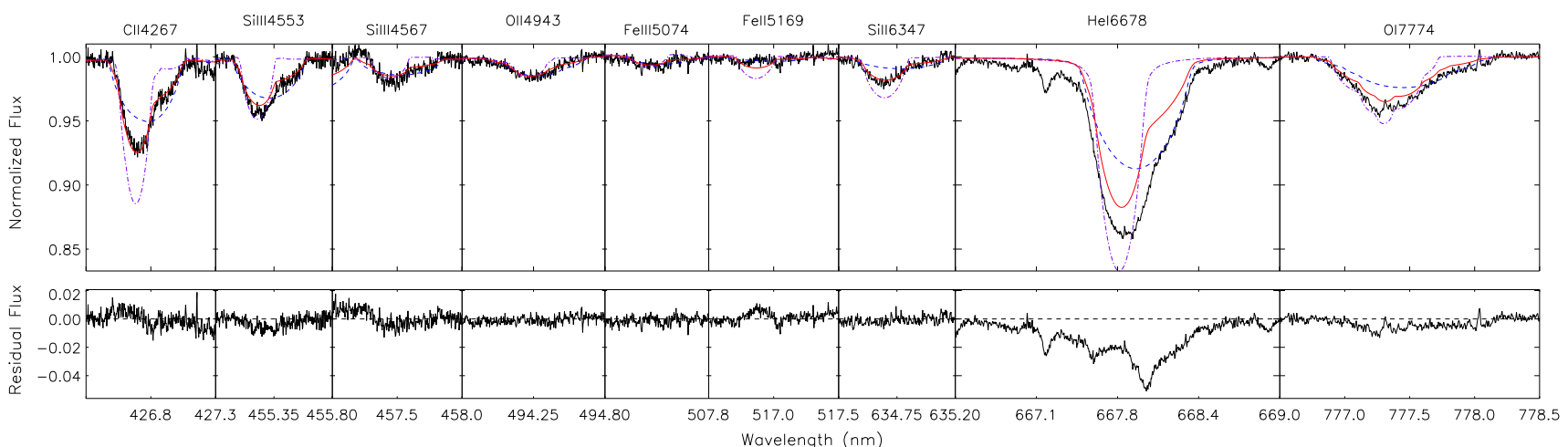

Figure 4. Comparison of the best-fit composite TLUSTY spectrum (red lines) to the observed mean spectrum obtained via co-addition of observations with similar RVs (black lines). Contributions from the primary (dashed blue) and secondary (dot-dashed purple) show the intrinsic flux from each component, i.e. not scaled by the luminosity ratio. The bottom panel show the residuals after subtraction of the model. Note the poor fit to He I $667.8 \mathrm{~nm}$, indicative of one of the stars being a He-strong star; this line was not used for parameter determination.
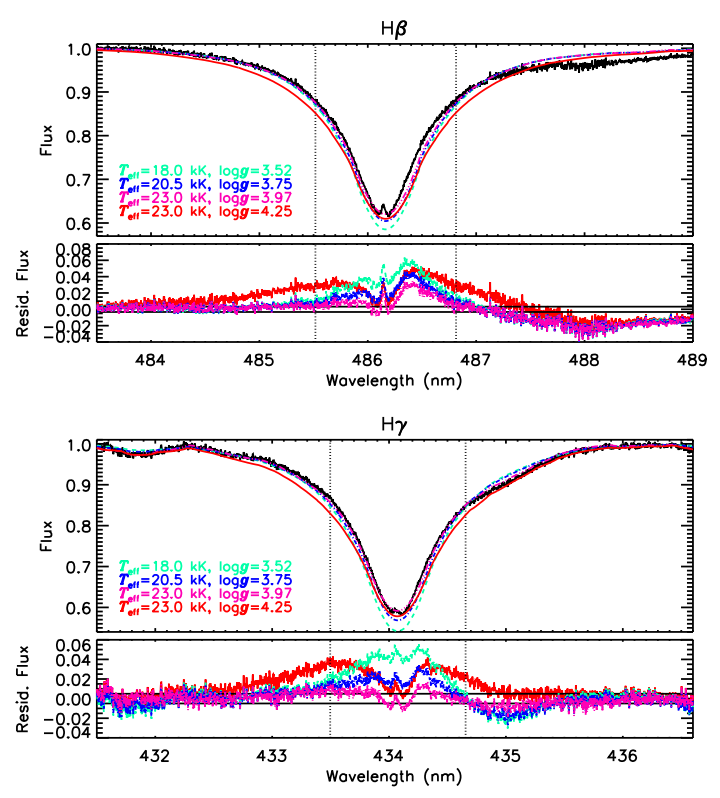

Figure 5. Single component fits to the $\mathrm{H} \beta$ and $\mathrm{H} \gamma$ lines. Vertical dotted lines indicate regions excluded from the fit. Note the flat residuals outside the exclusion regions; residuals inside are due to circumstellar emission. Legends indicate the $T_{\text {eff }}$ and $\log g$ of the corresponding models. Bottom sub-panels show the residual flux. Cyan, blue, and purple lines indicate best fit values for the given $T_{\text {eff }}$; the red line shows $\log g=4.25$ for comparison. Note the much deeper wings of the $\log g=4.25$ model in comparison to the observed spectrum.

(weaker) emission in the higher-numbered H Balmer lines. In order to mitigate the influence of this emission on the Balmer wings, a mean spectrum was created from the observations exhibiting the smallest amount of $\mathrm{H} \alpha$ emission. The mean spectra were obtained from merged and normalized spectral orders in the same fashion as described by Shultz et al. $(2019 \mathrm{~b})$. Fits were performed outside of $\pm 200 \mathrm{~km} \mathrm{~s}^{-1}$ i.e. excluding the rotationally broadened core of the primary. While the $\mathrm{H} \alpha$ emission can extend out to $\pm 700 \mathrm{~km} \mathrm{~s}^{-1}$, in the case of the low-emission mean spectrum the significant emission is contained within the $\pm 200 \mathrm{~km} \mathrm{~s}^{-1}$ exclusion
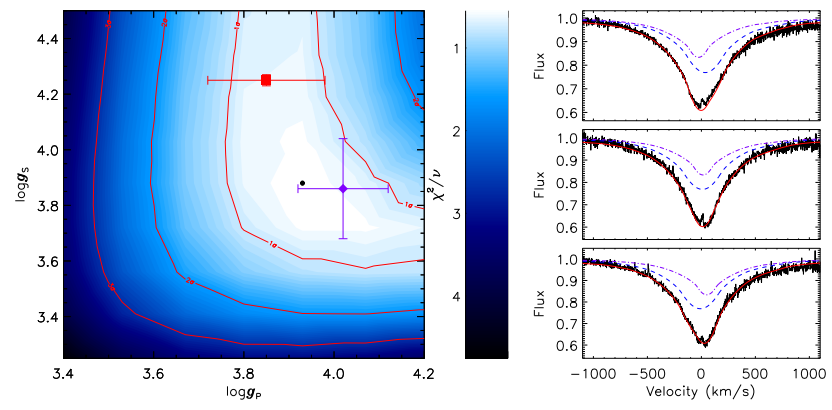

Figure 6. Two-component fit to $\mathrm{H} \beta$. The left panel shows the $\chi^{2}$ landscape (range indicated by colour bar), with the best-fitting model parameter indicated with black dots. Red contours indicate $\sigma$-levels. The diamond and square respectively indicate surface gravities inferred from main-sequence and pre-main-sequence evolutionary models (Fig. 7). Right panels show the fits to $\mathrm{H} \beta$, at (top to bottom), maximum primary RV, minimum RV separation, minimum primary RV (observed: black lines; dashed blue lines, primary; dot-dashed purple lines, secondary; solid red lines, combined flux).

range, as can be verified from the residuals in Fig. 5] Rotationally broadened synthetic spectra from the BSTAR2006 library were utilized for the fits, with $\chi^{2}$ minima being determined for 3 values of $T_{\text {eff }}$ spanning the range of $T_{\text {eff }}$ of the primary and secondary. Here we have made the assumption that a single-star model can yield reasonable results, given both the low amplitude of the RV variation and the similar $T_{\text {eff }}$ of the two stars. As can be seen from Fig. 5 , $\mathrm{H} \beta$ and $\mathrm{H} \gamma$ return similar values for $\log g, 3.68 \pm 0.22$ and $3.75 \pm 0.23$ (cgs) respectively.

We next performed a two-component fit to $\mathrm{H} \beta$ accounting for the contributions and RV variation of both stars. Here the same method was adopted as by Shultz et al. $(2019 \mathrm{~b})$, with the radius of the secondary constrained by the orbital mass ratio and $\log g$. As before, only observations exhibiting minimal emission, confined to the central $\pm 200 \mathrm{~km} \mathrm{~s}^{-1}$ region of the line, were utilized, and this velocity range was excluded from the fit. The results are shown in Fig. 6] and are consistent with the single-component fit: $\log g_{\mathrm{A}}=3.9_{-0.1}^{+0.3}$, and $\log g_{\mathrm{B}}=3.9_{-0.3}^{+0.5}$. 

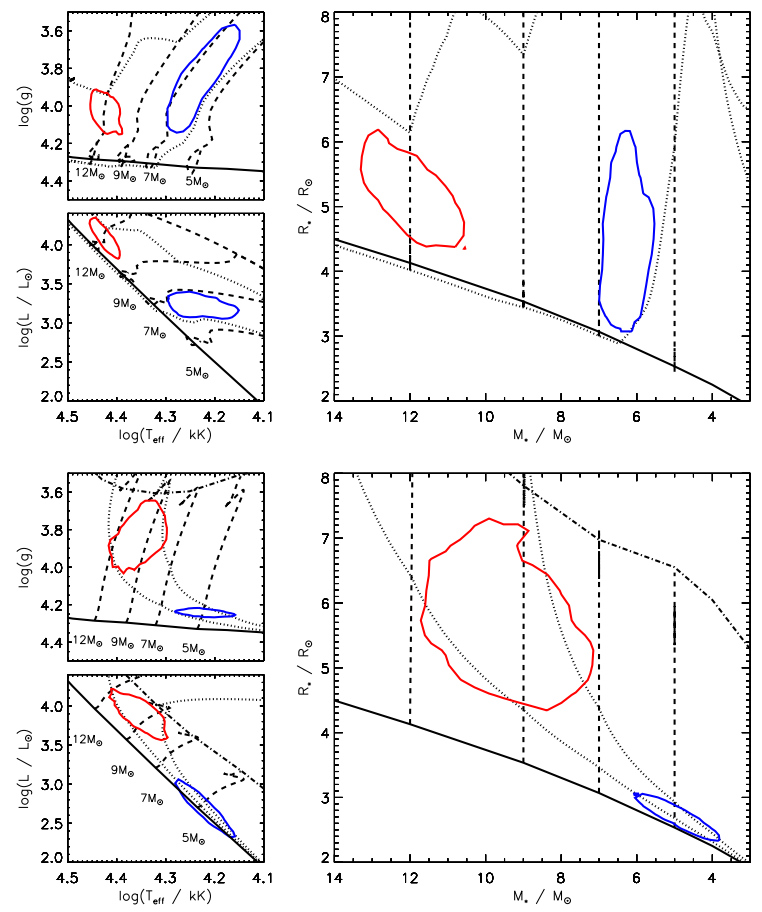

Figure 7. Stellar parameters inferred from MC sampling of the HRD using pre-MS models (Haemmerlé et al. 2019, top panels) and MS models (Ekström et al. 2012, bottom panels). Contours show $1 \sigma$ uncertainties (red: primary; blue: secondary). The solid line shows the Zero-Age Main Sequence; dashed and dotted lines respectively show evolutionary tracks and isochrones. For the PMS models, isochrones are shown for $\log t=5.4$ and 5.7 ; for the MS models, isochrones for $\log t=7.1$ and 7.5 are shown. A comparison between the $\log g$ values determined from evolutionary models to those measured via spectroscopic modelling is shown in Fig. 6

\subsection{Fundamental Parameters and Evolutionary Status}

W 601 has been reported as a pre-Main Sequence (PMS) star (Martavan et al. 2008; Alecian et al. 2008b). As its status as a classical Herbig Ae/Be star is doubtful due to the nature of its $\mathrm{H} \alpha$ emission (see $\S[$ and $\S 7$ ), it is not clear at this stage in the analysis whether the star is on the Main Sequence (MS) or the pre-MS, since its $\mathrm{H} \alpha$ emission does not appear to be from an accretion disk. Here we seek to determine whether the star is on the PMS or on the MS. The low measured surface gravity is potentially consistent with two scenarios: either the stars are on the PMS, or the primary has evolved towards the terminal age MS (TAMS).

As a first step, it is necessary to know whether or not W601 is a member of the young NGC 6611 cluster, since if it is a member this establishes an upper limit on the star's age, and additionally gives better constraints on the distance than are available from the Gaia parallax of the individual star. Cantat-Gaudin et al. (2018) identified W601 as a member of NGC 6611 with an $80 \%$ probability using Gaia DR2 parallaxes and proper motions (Gaia Collaboration et al. 2018). The star's DR2 parallax and proper motions are furthemore below the 3 and $5 \sigma$ rejection thresholds used by Kuhn et al. (2019) to determine cluster membership (respectively, differing by about $2.3 \sigma$ and $2.8 \sigma$ from the mean cluster values, using the smaller cluster uncertainty). The Gaia early Data Release 3 parallax and proper motions of W 601 are furthermore identical within uncertainty to the mean cluster values determined by Kuhn et al. (2019) on the basis of Gaia DR2 values. W 601's systemic velocity, $v_{0}=11.4 \pm 0.2 \mathrm{~km} \mathrm{~s}^{-1}$, is also consistent with the mean cluster radial velocity of $10 \pm 8 \mathrm{~km} \mathrm{~s}^{-1}$ determined from the VLT-FLAMES survey by Evans et al. (2005). We conclude that W 601 is very likely to be a member of NGC 6611 .

The mean Gaia parallax of the cluster is $\pi_{\mathrm{cl}}=0.57 \pm$ 0.04 mas, corresponding to a distance of $d=1740_{-120}^{+130} \mathrm{pc}$ (Kuhn et al. 2019). From isochrone fitting of the MS turnoff, the NGC 6611 cluster is between 0 and $6 \mathrm{Myr}$ in age according to de Winter et al. (1997), while Dufton et al. (2006) found an age of $3 \pm 1 \mathrm{Myr}$ using VLT-FLAMES data. Hillenbrand et al. (1993) found ages of $0.25 \mathrm{Myr}$ to $1 \mathrm{Myr}$ for young stellar objects (YSOs) below $8 \mathrm{M}_{\odot}$, and determined an average age of $2 \pm 1 \mathrm{Myr}$ for stars above $9 \mathrm{M}_{\odot}$. Getman et al. (2018) found the cluster's YSOs to be between 1 and 2.6 Myr. All studies seem to agree that the oldest stars in the cluster are no more than $6 \mathrm{Myr}$ in age, and that the cluster also contains ongoing star formation.

Measurements of extinction in NGC6611 are widely variable across the region, ranging from $A_{V}=1.7$ to 7.2 (e.g. Dufton et al. 2006; Guarcello et al. 2007; Maíz Apellániz \& Barbá 2018). There is furthermore evidence of a non-standard reddening law of between $R_{V}=3.5$ and 3.9 (e.g. Hillenbrand et al. 1993; Kumar et al. 2004; Guarcello et al. 2007; Maíz Apellániz \& Barbá 2018). To constrain the extinction and reddening of W601, we utilized the star's UBVJHK photometry (Høg et al. 2000; Wolff et al. 2007; Cutri et al. 2003), and de-reddened these using the IDL program FM_UNRED.PRO Fitzpatrick \& Massa 1999), with reddening $0<E(B-V)<1$ and reddening law $3<R_{V}<5$. The dereddened colours $U-B, B-V$, $V-J, V-H$ and $V-K$ were then compared to the intrinsic colours from the empirical PMS calibration determined by Pecaut \& Mamajek (2013). The best match was obtained for $E(B-V)=0.55$ and $R_{V}=4.37$, yielding $A_{V}=2.39$. The reddening law is steeper than obtained for other stars in the cluster, however fixing $R_{V}=3.5$ yields $E(B-V)=0.68$, with $A_{V}$ almost unchanged. These results are consistent with those obtained by previous examinations of the star (e.g. Kumar et al. 2004; Dufton et al. 2006).

In order to constrain the luminosity and the other fundamental stellar parameters, the binary Monte Carlo (MC) Hertzsprung-Russell Diagram (HRD) sampler described by Pablo et al. (2019) was utilized. This algorithm takes as priors the photometric properties of the system ( $V$ magnitude, extinction, parallax), the orbital mass ratio (Table 2), and the individual effective temperatures and surface gravities, and infers the luminosities, masses, ages, and radii via interpolation through evolutionary models under the assumption that the two components are coeval (but not interacting) (Bonnell \& Bate 1994). Bolometric corrections for both stars were obtained from the calibration of Nieva (2013). The $T_{\text {eff }}-\log g$ diagram is sampled with points drawn from gaussian distributions matching $T_{\text {eff }}$ and $\log g$ values of the two stars, and are probabilistically rejected if the inferred mass ratio, absolute $V$ mag, and age differ from target values drawn from gaussian distributions with standard devi- 
ations set respectively by the measured uncertainty in the mass ratio, the combined uncertainty in distance modulus and extinction, and an arbitrary tolerance in $\log t$. The results are shown in Fig. 7 and the parameters are given in Table 3

We determined stellar parameters using two sets of evolutionary models, the rotating MS models published by Ekström et al. (2012) (i.e., those with an initial rotation velocity of 0.4 of the critical value; bottom panels of Fig. 7), and the rotating PMS models developed by Haemmerlé et al. (2019) (top panels of Fig. 7), both of them calculated using the Geneva 1D stellar evolution code. The MS models start at the ZAMS, whereas the PMS models start at the birthline. For the PMS models an age tolerance of $\log t=0.15$ was adopted for the MC sampling, in order to reflect the possibility that very young stars might not be perfectly coeval; for the MS models a tolerance of 0.05 was used, ensuring any accepted points would lie on the same isochrone.

PMS models yield an age of $\log (t / \mathrm{yr})=5.6 \pm 0.1$, while the MS models yield an age of $\log t=7.3 \pm 0.2$. Only the age derived from PMS evolutionary models is consistent with the 6 Myr $(\log t=6.8)$ upper limit on the cluster age.

The MS models predict that $\mathrm{W} 601 \mathrm{~A}$ has a larger radius than W601 B, whereas the PMS models yield similar radii (compare the top and bottom right panels of Fig. 7). The ratio of radii from PMS MC parameter determination $\left(R_{\mathrm{A}} / R_{\mathrm{B}}=1.2 \pm 0.3\right)$ is consistent with results from spectral modelling $\left(R_{\mathrm{A}} / R_{\mathrm{B}}=1.1 \pm 0.1\right)$. Using MS models, the radii ratio is $R_{\mathrm{A}} / R_{\mathrm{B}}=2.1 \pm 0.3$, which is not consistent with the spectroscopy.

While the MC sampler initially draws from distributions in $\log g$ and $T_{\text {eff }}$ as determined from spectroscopic measurements, due to the various rejection criteria the posterior distributions of $\log g$ do not necessarily resemble the input distributions. MS models can only maintain coevality if $\log g_{\mathrm{A}}=3.85 \pm 0.12$ and $\log g_{\mathrm{S}}=4.24 \pm 0.02$, i.e. if the primary has evolved about halfway towards the TAMS, the secondary should still be very close to the zero-age MS (ZAMS). Conversely, PMS models yield $\log g_{\mathrm{A}}=4.06 \pm 0.10$ and $\log g_{\mathrm{B}}=3.86 \pm 0.18$. A comparison to the spectroscopic measurement of $\log g$ is provided on the $\chi^{2}$ map in Fig. 6] Both the MS and PMS results are consistent with the bestfit value within the $1 \sigma$ contours, although the PMS results are closest to the $\chi^{2}$ minima.

PMS models yield a better match to the measured surface gravities, radius ratio, and the age of the NGC 6611 cluster. We therefore conclude that the scenario with the greatest consistency is that both components of W 601 are still contracting towards the ZAMS. It is additionally notable that the $\mathrm{H} \alpha$ line displays nebular emission ( $(6)$, suggesting the system is still partially embedded in the nebula.

As can be seen in the top panels of Fig. 7 PMS models indicate that the ages of $\mathrm{W} 601 \mathrm{~A}$ and $\mathrm{B}$ differ by about $\log t=0.2$, or about $200 \mathrm{kyr}$ at the inferred age of the system. Reducing the age tolerance does not change this result; in fact an age tolerance of $\log t=0.15$ is the minimum necessary to keep the rejection rate reasonably small. This may indicate that the two components are not precisely coeval, but that the primary formed before the secondary.

From the stellar masses, the MC parameter analysis additionally yields an orbital inclination $i_{\text {orb }}=31.5 \pm 0.9^{\circ}$ and thus a semi-major axis $a=1.18 \pm 0.02 \mathrm{AU}$.

\section{MAGNETOMETRY}

In order to increase the $S / N$ of the line profile from which the magnetic field is measured, Least Squares Deconvolution (LSD; Donati et al. 1997; Kochukhov et al. 2010) profiles were extracted from the ESPaDOnS spectra. We used a line mask obtained with an 'extract stellar' request from the Vienna Atomic Line Database (VALD3; Piskunov et al. 1995; Rvabchikova et al. 1997; Kupka et al. 1999, 2000; Rvabchikova et al. 2015) for a $19 \mathrm{kK}$ solar metallicity star, with $\log g=3.8$, and a line depth threshold of 0.1. The mask was cleaned in the usual fashion in order to remove contamination from $\mathrm{H}$ Balmer, telluric, and interstellar lines (e.g. Shultz et al. 2018b). In addition to the intersellar Ca lines, W 601's spectrum contains several Diffuse Interstellar Bands (DIBs) which were also removed from the line mask. DIBs were identified by eye, including but not necessarily limited to lines with approximate wavelengths of $472 \mathrm{~nm}, 476 \mathrm{~nm}$, $496 \mathrm{~nm}, 523 \mathrm{~nm}, 540 \mathrm{~nm}, 541 \mathrm{~nm}, 549 \mathrm{~nm}, 551 \mathrm{~nm}, 554 \mathrm{~nm}$, $578 \mathrm{~nm}, 579 \mathrm{~nm}, 585 \mathrm{~nm}, 661 \mathrm{~nm}$, and $666 \mathrm{~nm}$.

Due to the large $v \sin i$ and low $S / N$ of the data, He lines were left in the mask. The resulting LSD profiles were scaled to a line depth of 0.1 , a mean wavelength of $500 \mathrm{~nm}$, and a mean Landé factor of 1.2. To further increase the perpixel $S / N$ a velocity pixel width of $7.2 \mathrm{~km} \mathrm{~s}^{-1}$ was used. A Tikhonov regularization factor of 0.2 was employed in order to suppress noise arising from the deconvolution process (Kochukhov et al. 2010). Following extraction, the LSD profiles were iteratively disentangled using the same procedure as adopted in $\S 3$ (e.g. González \& Levato 2006). The results are shown in Fig. 8. The Zeeman signature is confined within the line profile of $\mathrm{W} 601 \mathrm{~B}$, and tracks the radial velocity variation of this component; thus, it is W $601 \mathrm{~B}$ that hosts the magnetic field. Further magnetic analysis was therefore conducted using the disentangled Stokes $I$ profiles of W 601 B. Since the non-magnetic star only contributes noise to Stokes $V$, flux dilution does not affect magnetic measurements so long as the disentangled spectrum of the magnetic component is used (see e.g. Petit et al. 2019).

Observations were classified as definite, marginal, or non-detections (DD, MD, or ND) according to the False Alarm Probabilities (FAPs) measured inside the line profile, using the method and criteria described by Donati et al. (1992, 1997). The integration ranges used for measuring the FAP are shown in Fig. 8, and detection flags for individual stars are given in Table 1 Of the 27 total observations, 18 yield DDs, 2 MDs, and 7 NDs. FAPs measured from the null $N$ spectra are uniformly non-detections.

We measured the strength of the magnetic field via the disk-averaged longitudinal magnetic field (e.g. Mathys 1989; Wade et al. 2000), using the same integration ranges as used for measuring the FAP (i.e. about $\pm 120 \mathrm{~km} \mathrm{~s}^{-1}$ centred on the RV of $\mathrm{W} 601 \mathrm{~B})$. These measurements, as well as the $\left\langle N_{z}\right\rangle$ measurements obtained from the $N$ profiles, are given in Table 1 .

While the $N$ profiles are all NDs, the $\left\langle N_{z}\right\rangle$ measurements show a systemetic bias towards negative values. Close examination revealed that several of the Stokes $V$ and $N$ 


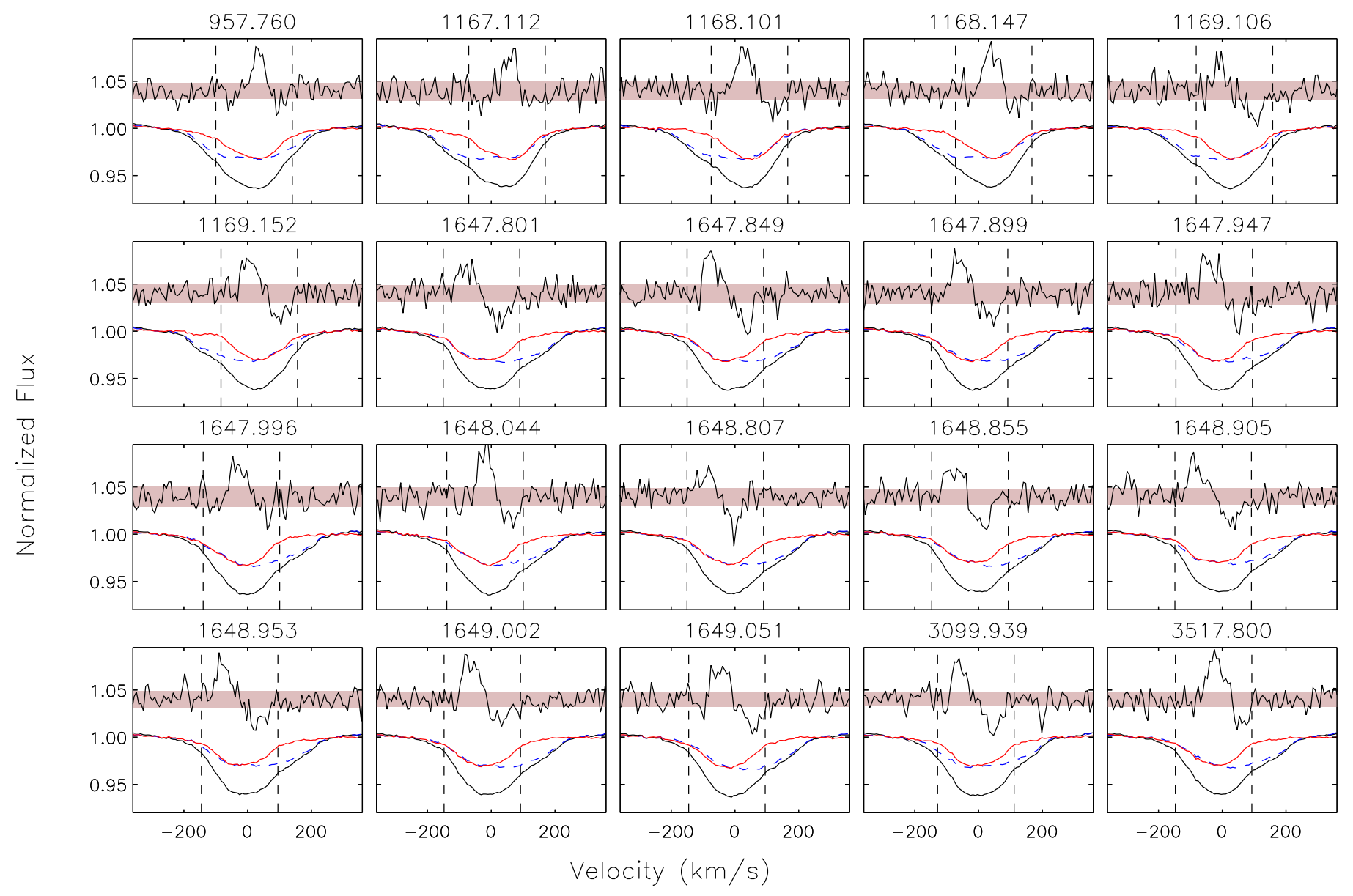

Figure 8. LSD profiles. Each panel is labelled with HJD-2453000. Only LSD profiles yielding at least a marginal detection in Stokes $V$ are shown. In each panel, the full Stokes $I$ profile is shown with black lines. The disentangled Stokes $I$ profiles of W $601 \mathrm{~A}$ and B are respectively shown with dashed blue and solid red lines. Above Stokes $I$ is the Stokes $V$ profile. The shaded region indicates the mean uncertainty in Stokes $V$. Vertical dashed lines indicate the integration limits used for measuring the FAP and $\left\langle B_{z}\right\rangle$. Note that Stokes $V$ is confined within the line profile of the secondary, and tracks its RV variation. It is therefore the secondary that is the magnetic star.

continua of several of the observations are offset from the expected value of 0 . This may be a consequence of the ratio of continuum flux in the two polarization beams changing as the retarder rotates (Bagnulo et al. 2012), likely exacerbated by the low $S / N$ of the observations. Re-adjusting the continua of the LSD Stokes $V$ and $N$ profiles to null corrected this issue, yielding $\left\langle N_{z}\right\rangle$ scattered evenly about 0 . The amplitude of $\left\langle B_{z}\right\rangle$ was slightly increased, although not outside of the error bars.

$\left\langle B_{z}\right\rangle$ ranges from about -1 to $+2 \mathrm{kG}$, with a mean uncertainty of $360 \mathrm{G}$. Even without knowing the rotation period or magnetic configuration, the maximum value of $\left\langle B_{z}\right\rangle$ indicates that the surface magnetic dipole strength $B_{\mathrm{d}}$ must be at least $7 \mathrm{kG}$ (under the simplest assumption of a dipolar surface magnetic field, in which case the lower limit on $B_{\mathrm{d}}$ is about $3.5 \times$ the maximum value of $\left.\left\langle B_{z}\right\rangle\right)$. Since both positive and negative values of $\left\langle B_{z}\right\rangle$ are measured, both magnetic poles must come into view over the course of a rotational cycle.

\section{LINE PROFILE VARIABILITY}

W601 was originally classified as a Herbig Be star on the basis of its youth and $\mathrm{H} \alpha$ emission Martavan et al. 2008; Alecian et al. 2008b). T Tauri stars and Herbig Ae/Be stars generally show variable emission in multiple lines, not only $\mathrm{H} \alpha$ (Mendigutía et al. 2011a). The morphologies and variability patterns of the line emission of $\mathrm{T}$ Tauri stars are explained by the magnetospheric accretion model (Bouvier et al. 2007), in which the inner disk material is locked to and therefore in corotation with the star's magnetic field, leading to the formation of a gap between the photosphere and the inner rim of the disk. Linear $\mathrm{H} \alpha$ spectropolarimetry confirms the existence of this gap in both $\mathrm{T}$ Tauri stars and Herbig Ae stars (Vink et al. 2005), and the line emission of at least one magnetic Herbig Ae star is consistent with magnetospheric accretion (Schöller et al. 2016). However, the line emission of early-type Herbig Be stars is generally not consistent with magnetospheric accretion (Mendigutía et al. 2011b; Wichittanakom et al. 2020), which is not surprising given the very low incidence of detected magnetic fields in this population. 

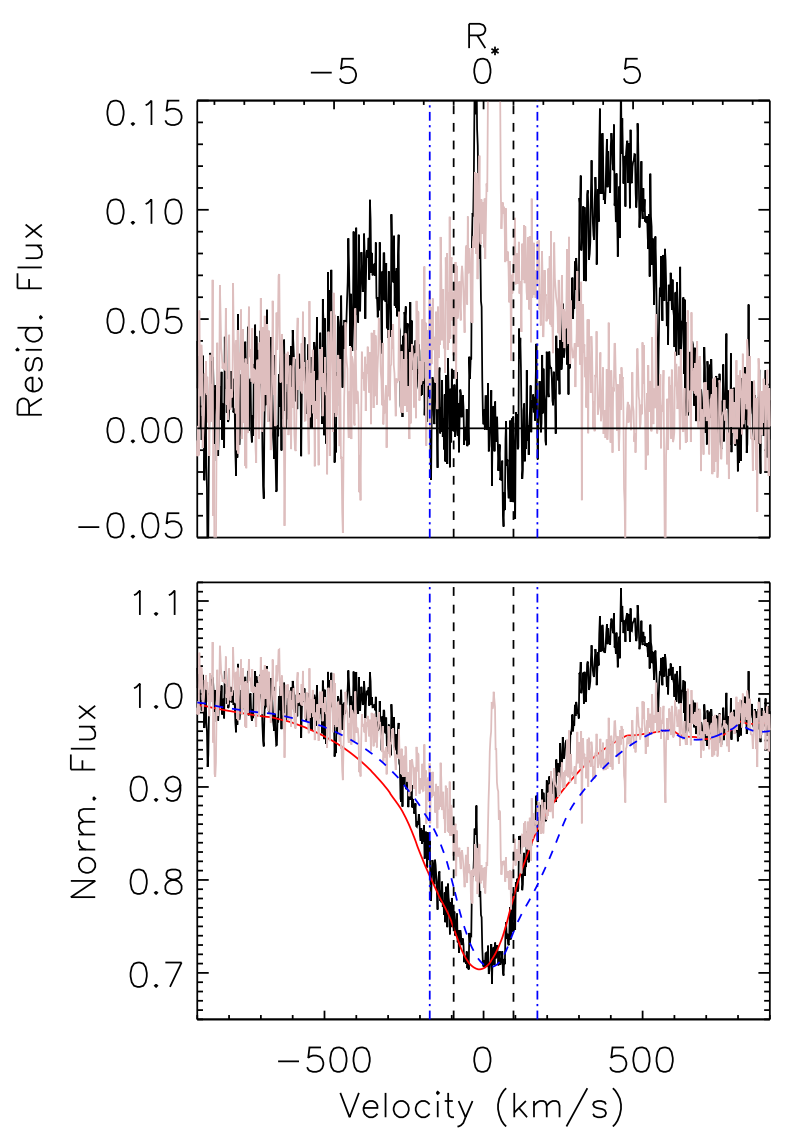

Figure 9. Bottom: H $\alpha$ profiles at minimum emission (grey) and maximum emission (black), with respective synthetic binary profiles (dashed blue, solid red). Dashed black vertical lines indicate $\pm v \sin i$ for $\mathrm{W} 601 \mathrm{~B}$; dot-dashed blue vertical lines indicate $\pm R_{\mathrm{K}}$. The profiles have been shifted to the rest-frame of the secondary. The thin emission feature in the centre of the line is nebular emission. Top: Residual flux after subtracting synthetic from observed spectra. The double-humped emission, peaking at several times $v \sin i$, is a characteristic signature of a centrifugal magnetosphere. Note that the top axis is in units of stellar radii, reflecting the assumption that the emission is produced by magnetically confined material in corotation with the star.

The presence of a strong magnetic field and evidently rapid rotation may indicate that $\mathrm{W} 601$ may be the first Herbig $\mathrm{Be}$ star with $\mathrm{H} \alpha$ emission consistent with magnetospheric accretion. However, the absence of the expected emission in other lines (Mendigutía et al. 2011a) suggests that the star's $\mathrm{H} \alpha$ profile may instead be consistent with an origin in a wind-fed 'Centrifugal Magnetosphere' (CM; Landstreet \& Borra 1978; Petit et al. 2013), since rapidly rotating, strongly magnetic early B-type stars almost invariably possess CMs detectable in $\mathrm{H} \alpha$ (Shultz et al. 2019d).

Inspection of the $\mathrm{H} \alpha$ line indicates that the morphology of the $\mathrm{H} \alpha$ emission is consistent with a CM, i.e. a double-humped emission profile with the peak emission occuring at velocities equal to several times the projected rotational velocity $v \sin i$, as can be seen in Fig. 9. This shape arises due to rigid corotation of magnetically confined plasma with the photospheric magnetic field, which results in the confined plasma rotating at velocities that increase linearly with distance from the star. Beyond the
Kepler corotation radius $R_{\mathrm{K}}$, centrifugal support is stronger than gravity and gravitational infall of the confined plasma is prevented (e.g. ud-Doula et al. 2008). This leads to an accumulation of material above $R_{\mathrm{K}}$, and a cavity below $R_{\mathrm{K}}$, thereby giving rise to the double-humped morphology (e.g. Townsend \& Owocki 2005). A Keplerian disk seen edge-on can also produce a double-humped emission profile, however the crucial difference with $\sigma$ Ori E-type emission is that the emission of a Keplerian disk is confined inside $\pm v \sin i$.

To analyze the time variability of $\mathrm{H} \alpha$ we measured its EWs. We utilized the individual Stokes $I$ spectra in order to maximize the size and time-resolution of the dataset (i.e. 108 spectra). EWs were measured in the red half of the line, from $+v \sin i$ to the red edge of emission at $+700 \mathrm{~km} \mathrm{~s}^{-1}$, and in the blue half of the line between the blue edge of emission at $-700 \mathrm{~km} \mathrm{~s}^{-1}$ and $-v \sin i$. The core of the line was excluded so as to avoid contaminating the measurements with nebular emission (Fig. 9). To correct for the EW variation due to the radial velocity variations of the two components, for each spectrum we calculated synthetic binary spectra in the same fashion as was done for $\mathrm{H} \beta$ in determining $\log g$ (Fig. 6), using the best-fit parameters for each star and a radius ratio of $R_{\mathrm{A}} / R_{\mathrm{B}}=1.1$ as inferred from spectroscopic modelling (Fig. 4). The EWs of the model spectra were measured within the same integration ranges, and subtracted from the EWs of the data. These EWs are used to determine the rotational period below in $\S 7.1$. Line profile variations are examined in greater detail in the context of the magnetospheric analysis in $\S 9.1$

Magnetic hot stars typically have surface chemical abundance spots that lead to rotational modulation of EW measurements from photospheric line profiles. We examined the He I $667.8 \mathrm{~nm}$, Si III $455.4 \mathrm{~nm}$, and C II $426.7 \mathrm{~nm}$ lines, choosing these lines on the basis of being relatively strong and isolated. The EWs of these line were measured from individual Stokes $I$ subexposures across the full line width, as inferred by eye, with the individual spectra renormalized using a linear fit to adjacent continuum regions. There is no statistically significant variability in either Si III or C II (the reduced $\chi^{2}$ for the null assumption of no variation about the mean value being in both cases about unity). He I, however, exhibits statistically significant variation, and this line was also included in the period analysis in $\S 7.1$.

\section{ROTATION}

\subsection{Period determination}

Magnetic hot stars generally exhibit spectropolarimetric and spectroscopic variations modulated precisely by the rotation period, with no inter-cycle variations i.e. the variability is perfectly regular. Therefore, the rotation period can be determined independently using multiple diagnostics. The period analysis of individual diagnostics $\left(\left\langle B_{z}\right\rangle, \mathrm{H} \alpha\right.$ and He I $667.8 \mathrm{~nm} \mathrm{EWs)}$ is described in the following, with 1.131805(6) d from $\mathrm{H} \alpha$ EWs being adopted as the most likely rotational period.

As a first attempt to determine the rotation period the $\left\langle B_{z}\right\rangle$ measurements were analyzed using PERIOD04. The resulting periodogram is shown in the top panel of Fig. 10. The shaded region indicates the range of physically plausible rotation periods, with the lower bound set by the breakup 

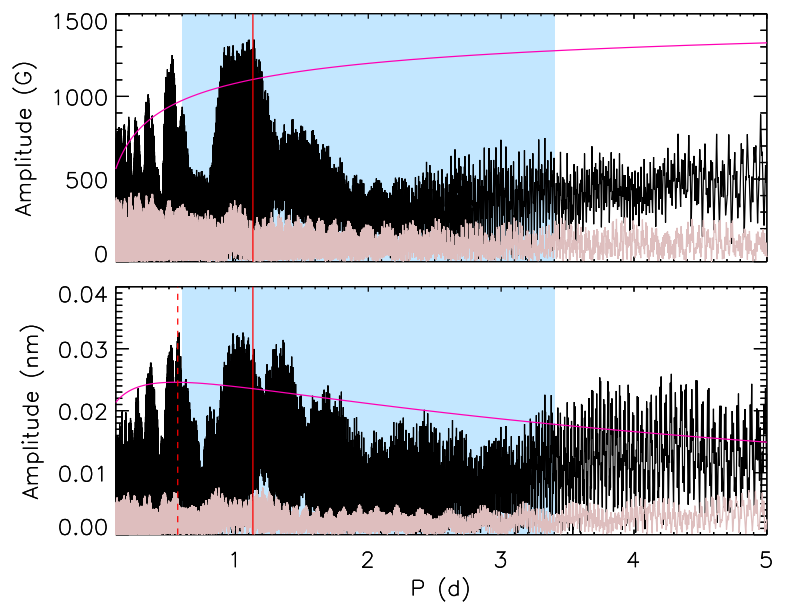

Figure 10. Periodograms for $\left\langle B_{z}\right\rangle$ (top) and H $\alpha$ EWs (bottom). Shaded regions indicate the physically plausible range of periods (see text). Periodograms are shown in black. The $3 \sigma$ noise level is shown by purple curves. The adopted rotation period is indicated with a solid red line. For the $\mathrm{H} \alpha$ periodogram, red dashed lines indicate rotational harmonics. Grey periodograms are after prewhitening with the rotation period (top) or the rotation period and its harmonics (bottom).

velocity and the upper bound set by $R_{*}$ and $v \sin i$. Within this window, the highest peak is at 1.13980(3) d (with the uncertainty in the least significant digit given in parentheses), with a $S / N$ of 9 , i.e. the period is above the threshold of 4 usually adopted for formal significance (Breger et al. 1993; Kuschnig et al. 1997). However, there are numerous other nearby peaks with a similar amplitude, and it is not possible to distinguish between them based purely on the coherence of the phase variation.

We also analyzed the EWs described in $\S 6$ using PERIOD04. The H $\alpha$ periodogram is shown in the bottom panel of Fig. 10. There is a single strong peak at about $1.15 \mathrm{~d}$, consistent with the forest of peaks obtained for $\left\langle B_{z}\right\rangle$. However, the strongest signal is at $0.56589 \mathrm{~d}$, very close to the first harmonic of the period identified in $\left\langle B_{z}\right\rangle$. This is typical of the double-wave EW variations produced by the CMs of stars in which both magnetic poles are visible during a rotation cycle, as is the case with W 601 B (e.g. Landstreet \& Borra 1978; Shultz et al. 2020). Under the presumption that this is one-half of the true rotation period, we fit the period and this harmonic with PERIOD04 in order to obtain a period of $1.13179(1)$ d. The $S / N$ of the rotational period and its first harmonic are respectively 7 and 12 . If EWs measured from the red half of the line $(\S[6)$ are analyzed in isolation where the emission strength is at a maximum and the amplitude of variation is the largest - the respective $S / N$ of the rotation period and its first harmonic are 27 and 29, with $P_{\text {rot }}=1.131805(6) \mathrm{d}$. Analyzing the blue $\mathrm{H} \alpha$ EWs in isolation does not yield useful results. The strongest peak in the periodogram is at about $0.558783(6) \mathrm{d}$, qualitatively consistent although formally inconsistent with the first harmonic of the period obtained from the red EWs; however, the $S / N$ of this period is not high (5.6). We adopt the period obtained from the red EWs as the most precise, and the most likely to be correct.

For He I $667.8 \mathrm{~nm}$, the strongest peak is at 1.09099(2)

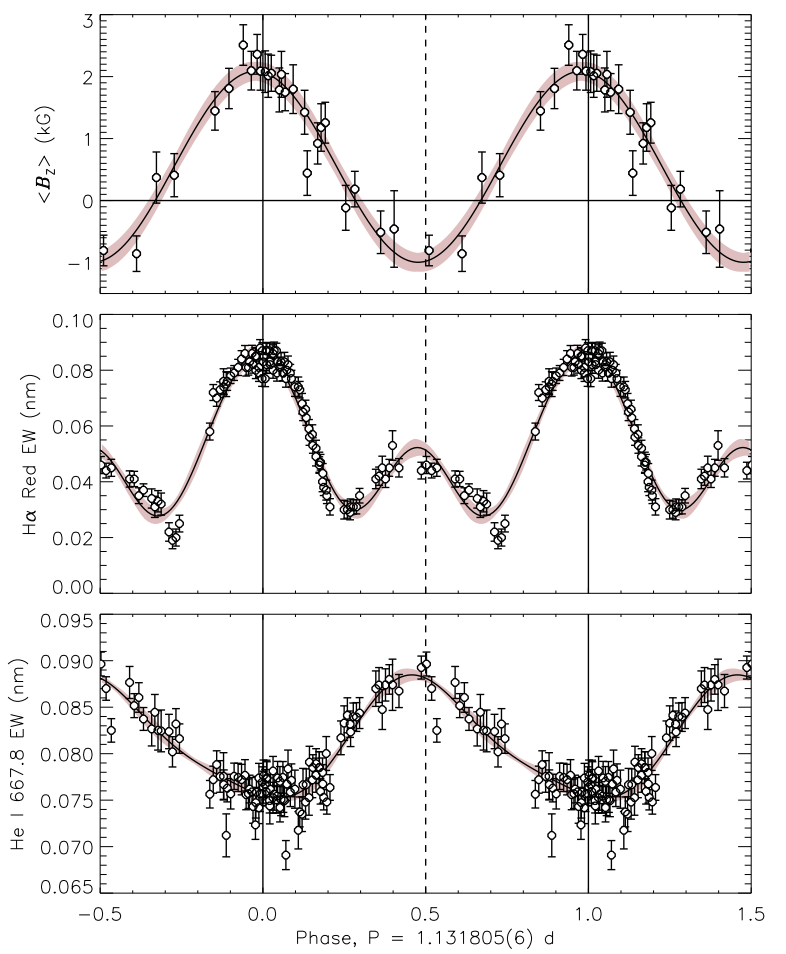

Figure 11. $\left\langle B_{z}\right\rangle$ (top), red $\mathrm{H} \alpha$ emission EW (middle), and He I $667.8 \mathrm{~nm}$ EW (bottom), phased with the rotation period. Curves and grey shaded regions show harmonic fits to the data and $1 \sigma$ uncertainties. Solid and dashed vertical lines indicate magnetic extrema. EWs were obtained from individual Stokes $I$ subexposures. All data have been sigma-clipped to remove the noisiest observations. $\mathrm{H} \alpha$ emission peaks occur at the extrema of $\left\langle B_{z}\right\rangle$, as expected for rotationally modulated magnetospheric emission.

d. However, if the He I EWs are phased with the $\mathrm{H} \alpha$ period, PERIOD04 returns a higher amplitude (0.006 nm vs. 0.005 $\mathrm{nm})$ and a higher $S / N$ (11.5 vs. 9.8). Since $\mathrm{H} \alpha$ yields a higher $S / N$, the slightly different period returned by He I $667.8 \mathrm{~nm}$ is probably a consequence of the lower $S / N$ of the variation of this line (the semi-amplitude of variation in this line being less than $3 \times$ the mean error bar).

\subsection{Rotational Modulation}

$\left\langle B_{z}\right\rangle, \mathrm{H} \alpha \mathrm{EWs}$, and He I $667.8 \mathrm{~nm}$ EWs are shown phased with $P_{\text {rot }}$ in Fig. 11] using $J D 0=2453957.4(2)$ as determined by fitting a sinusoid to $\left\langle B_{z}\right\rangle$ and determining the $\left\langle B_{z}\right\rangle$ maximum one cycle before the first observation. The reduced $\chi^{2}$ of a first-order sinusoidal fit to $\left\langle B_{z}\right\rangle$ is 1.3 , indicating that a sinusoid is a good fit and that $\left\langle B_{z}\right\rangle$ can therefore be reproduced by a simple tilted dipole, as is the case for most magnetic early-type stars (e.g. Shultz et al. 2018b; Kochukhov et al. 2019).

The He I EWs phase coherently with the rotational period (Fig. 11, bottom), with the extrema of the He I EW curve corresponding to the $\left\langle B_{z}\right\rangle$ extrema. The He variability pattern suggests that the strongest He abundance spot is at the negative magnetic pole. Coherent EW variation is 
Table 4. Rotational, magnetic, and magnetospheric parameters for W 601 B (see text for definitions.)

\begin{tabular}{lc}
\hline \hline Parameter & Value \\
\hline$P_{\text {rot }}(\mathrm{d})$ & $1.13178 \pm 0.00001$ \\
$T_{0}(\mathrm{HJD})$ & $2453957.4 \pm 0.2$ \\
$i_{\text {rot }}\left(^{\circ}\right)$ & $31_{-3}^{+5}$ \\
$v_{\text {eq }}(\mathrm{kms})$ & $161_{-9}^{+33}$ \\
$W$ & $0.24_{-0.01}^{+0.09}$ \\
$R_{\mathrm{p}} / R_{\mathrm{e}}$ & $0.971_{-0.03}^{+0.003}$ \\
$R_{\mathrm{K}}\left(\mathrm{R}_{*}\right)$ & $2.1_{-0.2}^{+0.2}$ \\
\hline$B_{0}(\mathrm{kG})$ & $0.54 \pm 0.07$ \\
$B_{1}(\mathrm{kG})$ & $1.55 \pm 0.09$ \\
$\beta\left({ }^{\circ}\right)$ & $79_{-3}^{+1}$ \\
$B_{\mathrm{d}}(\mathrm{kG})$ & $11_{-1}^{+3}$ \\
\hline $\log \left(\dot{M}^{+3} / \mathrm{M}_{\odot} / \mathrm{yr}\right)$ & $-9.5 \pm 0.1$ \\
$v_{\infty}(\mathrm{km} / \mathrm{s})$ & $1120 \pm 60$ \\
$\log \eta_{*}$ & $6.0 \pm 0.2$ \\
$R_{\mathrm{A}}\left(\mathrm{R}_{*}\right)$ & $31_{-2}^{+5}$ \\
$\log R_{\mathrm{A}} / R_{\mathrm{K}}$ & $1.2 \pm 0.1$ \\
$\log \left(B_{\mathrm{K}} / \mathrm{G}\right)$ & $2.7 \pm 0.2$ \\
$\log \left(\tau_{\mathrm{J}} / \mathrm{yr}\right)$ & $6_{-0.1}^{+0.3}$ \\
$\log \left(t_{\mathrm{S}, \mathrm{max}} / \mathrm{yr}\right)$ & $5.8_{-0.1}^{+0.5}$ \\
\hline \hline
\end{tabular}

notable in the case of a PMS star, as it suggests that surface chemical abundance patches form almost immediately in the photospheres of magnetic hot stars.

The rotational modulation of $\mathrm{H} \alpha$ is examined in detail in $\S 9.1$

\section{MAGNETIC MODELLING}

The rotationally magnetic magnetic variability of hot stars is described using the Oblique Rotator Model (ORM; e.g. Stibbs 1950), in which the sinusoidal variation in $\left\langle B_{z}\right\rangle$ of a dipolar magnetic field rotating in the plane of the sky is parameterized with the inclination $i_{\text {rot }}$ of the rotational axis from the line of sight, the tilt angle $\beta$ of the magnetic axis from the rotational axis, and the strength of the magnetic dipole at the stellar surface $B_{\mathrm{d}}$.

To determine W 601 B's rotational, ORM, and magnetospheric parameters, we utilized the Monte Carlo (MC) Hertzsprung-Russell Diagram (HRD) sampler described by Shultz et al. (2019d). The MC sampler combines information about a star's observed atmospheric, magnetic, and rotational properties, together with ancillary information such as e.g. the age of its parent cluster, with evolutionary models in order to infer fundamental stellar parameters, Oblique Rotator Model (ORM) parameters, rotational parameters, and magnetospheric parameters. These are given in Table 4

As inputs we used the stellar parameters obtained above (Fig. 7 Table 3), the star's rotational period, and $v \sin i$ to obtain $i_{\text {rot }}=31_{-3}^{\circ+5}$. This is similar to the orbital axis inclination $i_{\text {orb }}=31.5 \pm 0.9^{\circ}$, i.e. the spin and orbital axes of the system are aligned or nearly aligned. The sinusoidal fitting parameters to $\left\langle B_{z}\right\rangle=B_{0}+B_{1} \sin (\phi+\Phi)$ are $B_{0}=540 \pm 70 \mathrm{G}, B_{1}=1550 \pm 90 \mathrm{G}$, and phase offset $\Phi=1.67 \pm 0.09 \mathrm{rad}$ (from the model shown in the top panel of Fig. 11). From the geometrical relations given by Preston
(1967, 1974), and with the maximum measured value of $\left\langle B_{z}\right\rangle$ being $\left\langle B_{z}\right\rangle_{\max }=2509 \pm 327 \mathrm{G}$, the magnetic axis obliquity angle is then $\beta=79_{-3}^{\circ+1}$ and the surface magnetic dipole strength is $B_{\mathrm{d}}=11_{-1}^{+3} \mathrm{kG}$ (using a linear limb darkening coefficient $\epsilon=0.4$ from line-blanketed NLTE model spectra; Reeve \& Howarth 2016).

Another means of determining W 601 B's ORM parameters is via direct modelling of Stokes $V$ using a version of the Bayesian inference method described by Petit \& Wade (2012) modified to include rotational phase information. The results of this fit are shown compared to observations in Fig. 12 Direct modelling of Stokes $V$ produces a best fit for $i_{\text {rot }}=54_{-5}^{+20^{\circ}}, \beta=60_{-10}^{+5^{\circ}}$, and $B_{\mathrm{d}}=6.2_{-0.4}^{+0.8} \mathrm{kG}$, where the uncertainties correspond to the $68.7 \%$ credible regions. These values differ substantially from the values inferred from $\left\langle B_{z}\right\rangle$ and the stellar parameters, although values of $B_{\mathrm{d}}$ comparable to those inferred from $\left\langle B_{z}\right\rangle$ can be accommodated within the $95.4 \%$ credible region. Note that, unlike $\left\langle B_{z}\right\rangle$ fitting, direct modelling of the Stokes $V$ profile does not constrain $i_{\text {rot }}$ from $R_{*}, v \sin i$, and $P_{\text {rot }}$. The smaller value of $B_{\mathrm{d}}$ found by modelling Stokes $V$ is due to the larger value of $i_{\text {rot }}: i_{\text {rot }}<45^{\circ}$ is excluded by the Stokes $V$ fits, whereas $i_{\text {rot }}>30^{\circ}$ is excluded by the star's radius and rotational properties. The Stokes $V$ model and the $\left\langle B_{z}\right\rangle$ model agree well near magnetic maximum at phase 0 . However, the $\left\langle B_{z}\right\rangle$ model predicts a crossover signature with a larger amplitude as the magnetic equator comes into view near phases 0.2 and 0.7. A possible reason for this discrepancy may be that, notwithstanding the reasonable fit of a dipole model to $\left\langle B_{z}\right\rangle$, the magnetic field is not purely dipolar but instead a 'distorted dipole' (the most common toplogy revealed by Zeeman Doppler Imaging; Kochukhov et al. 2019). Supporting this supposition, neither set of ORM parameters provides a faithful reproduction of Stokes $V$ at all phases.

An alternative explanation for the tension between the ORM parameters inferred from $\left\langle B_{z}\right\rangle$ and those obtained via direct modelling of Stokes $V$ is systematic error in W 601 B's stellar parameters. If $\mathrm{W} 601 \mathrm{~B}$ is actually at the ZAMS, then assuming a mass of $5.7 \pm 0.3 \mathrm{M}_{\odot}$ its minimum possible radius is $R_{*}=2.9 \pm 0.1$. This results in $i=44 \pm 3^{\circ}, \beta=75 \pm 3^{\circ}$, and $B_{\mathrm{d}}=7.9_{-0.7}^{+1} \mathrm{kG}$, very similar to the values found via modelling of Stokes $V$. However, this would require $\log g=$ 4.3 , in which case W601 A would need to have a surface gravity of 3.7 (left panel of Fig. 6). In this case, the age of the system would need to be about $\log t=7.2$, which is much older than the NGC 6611 cluster $(\log t \sim 6.75)$. W $601 \mathrm{~A}$ would also have a much larger radius than $\mathrm{W} 601 \mathrm{~B}$, which is inconsistent with spectroscopic modelling, which instead indicate their radii are almost identical.

\subsection{Magnetic Constraints on the Primary}

The line profiles of the two stellar components are blended in all observations, making it difficult to constrain the magnetic properties of W601 A. To do this, we subtracted the model fits to Stokes $V$ obtained via Bayesian inference (Fig. 12) from the observed Stokes $V$ profiles, yielding composite LSD profiles consisting of the disentangled Stokes $I$ profiles of W $601 \mathrm{~A}$ and the residual Stokes $V$ profiles. We measured FAPs and $\left\langle B_{z}\right\rangle$ in the usual fashion, with an integration range of $\pm 220 \mathrm{~km} \mathrm{~s}^{-1}$ around the rest frame of $\mathrm{W} 601 \mathrm{~A}$. All of the profiles are non-detections, indicating that, despite 


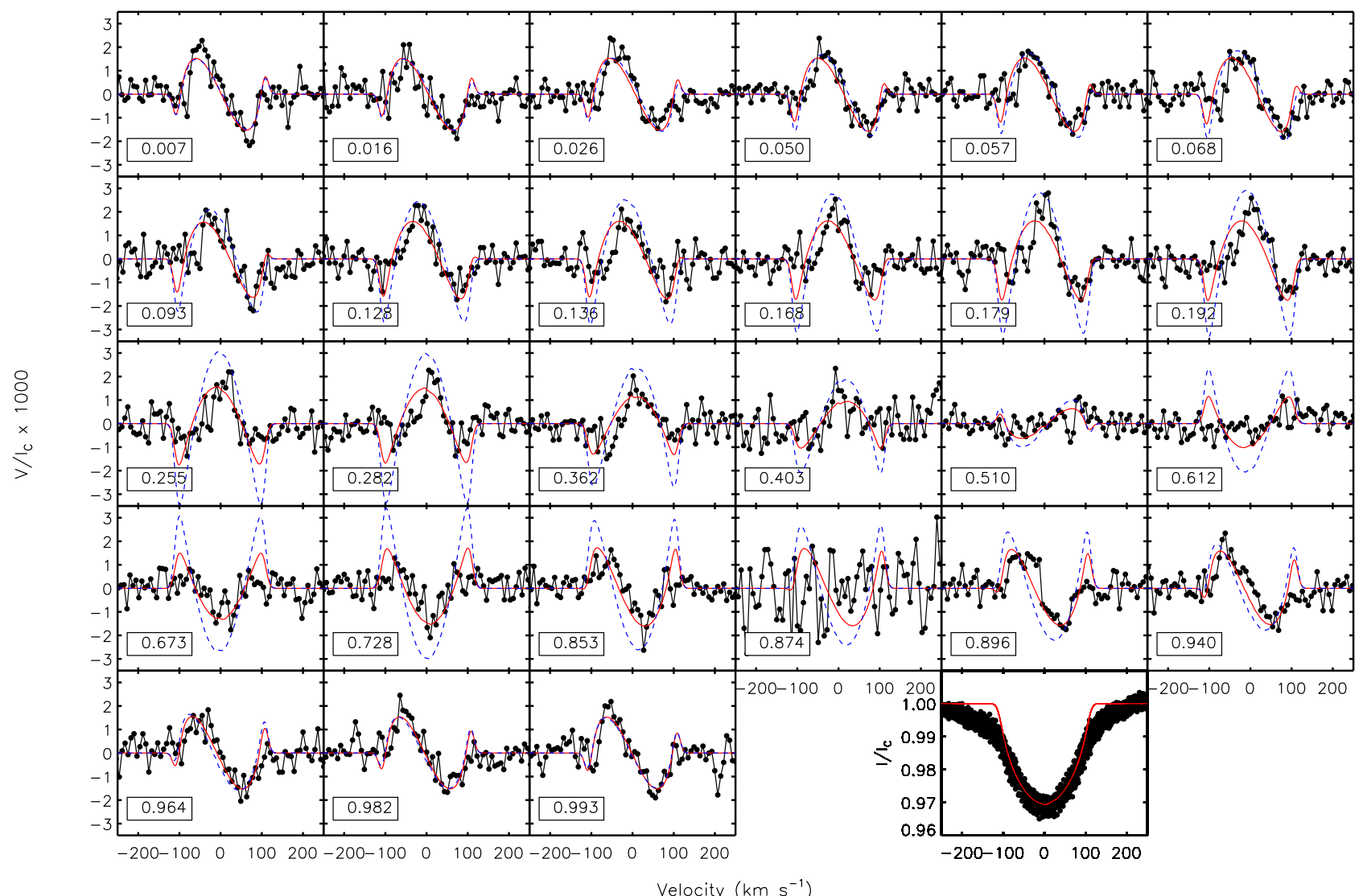

Figure 12. Observed LSD Stokes $V$ profiles (black circles) compared to synthetic Stokes $V$ profiles inferred from the $\left\langle B_{z}\right\rangle$ curve model (dashed blue) and from direct Bayesian modelling of Stokes $V$ (solid red). Rotational phases are indicated in boxes in the upper left corner of each panel. The bottom right panel shows the overplotted distenangled Stokes $I$ profiles of W 601 B, as compared to the synthetic Stokes $I$ profile (red line) used to model Stokes $V$. Neither model provides a fully satisfactorily reproduction of the Stokes $V$ variation.

the imperfections in the fit to Stokes $V$, the residuals are not consistent with statistically significant departures from null polarization. The mean $\left\langle B_{z}\right\rangle$ error bar is $450 \mathrm{G}$, and the root-mean-square $\left\langle B_{z}\right\rangle$ is $365 \mathrm{G}$, further confirming that the Zeeman signature was successfully removed.

We then analyzed the profiles using the same Bayesian inference engine used to infer ORM parameters for W $601 \mathrm{~B}$, with the difference that, since $P_{\text {rot }}$ is unknown, rotational phase was treated as a nuisance parameter Petit \& Wade 2012). The Stokes $V$ profiles yielded upper limits on $B_{\mathrm{d}}$ at $68.3 \%, 95.4 \%, 99.0 \%$, and $99.7 \%$ credible regions of $306 \mathrm{G}$, $826 \mathrm{G}, 1704 \mathrm{G}$, and $2832 \mathrm{G}$ respectively. The corresponding upper limits obtained from $N$ are comparable, respectively 279 G, 779 G, $1590 \mathrm{G}$, and $2640 \mathrm{G}$.

Any possible magnetic field possessed by W $601 \mathrm{~A}$ is less than W 601 B's by a factor of at least 2 with $99.7 \%$ credibilty. With $68.3 \%$ credibility its magnetic field is less than 300 $\mathrm{G}$, which is the approximate critical magnetic field strength necessary for the magnetic field to maintain stability against rotational or convective instabilities (Aurière et al. 2007; MacDonald \& Petit 2019; Jermvn \& Cantiello 2020). Fossil magnetic fields weaker than $300 \mathrm{G}$ are exceptionally uncommon (Aurière et al. 2007; Sikora et al. 2019b; Shultz et al. $2019 \mathrm{~d}$ ), and those magnetic fields that have been detected below this threshold are usually either in evolved stars (e.g.
Fossati et al. 2015; Neiner et al. 2017; Martin et al. 2018) or ultra-weak fields (on the order of $\sim 0.1-10 \mathrm{G}$ ) as found in Vega (Lignières et al. 2009; Petit et al. 2010), or in Am stars such as Sirius (Petit et al. 2011), Alhena (Blazère et al. 2016, 2020), and others. While no ultra-weak field has yet been detected in a B-type star, the similarly of the magnetic properties of A and B-type stars (Shultz et al. 2019d) suggests that the 'magnetic desert' between the ultra-weak fields and fossil magnetic fields likely persists across the entire upper main sequence. Since the upper limit on W 601 A's magnetic field is comparable to the critical magnetic field strength, any potential magnetic field is likely to be on the order of a few tens of $\mathrm{G}$ or less.

\section{DISCUSSION}

\subsection{Magnetosphere}

Rapidly rotating, strongly magnetic B-type stars frequently display $\sigma$ Ori E variability originating in circumstellar magnetospheres (Landstreet \& Borra 1978). H $\alpha$ (Fig. 11 middle) shows a double-wave variation, with the strongest of the maxima corresponding to the $\left\langle B_{z}\right\rangle$ maximum and the weaker local maximum corresponding to the $\left\langle B_{z}\right\rangle$ minimum (vertical lines in Fig. 11). This double-wave EW variation is 


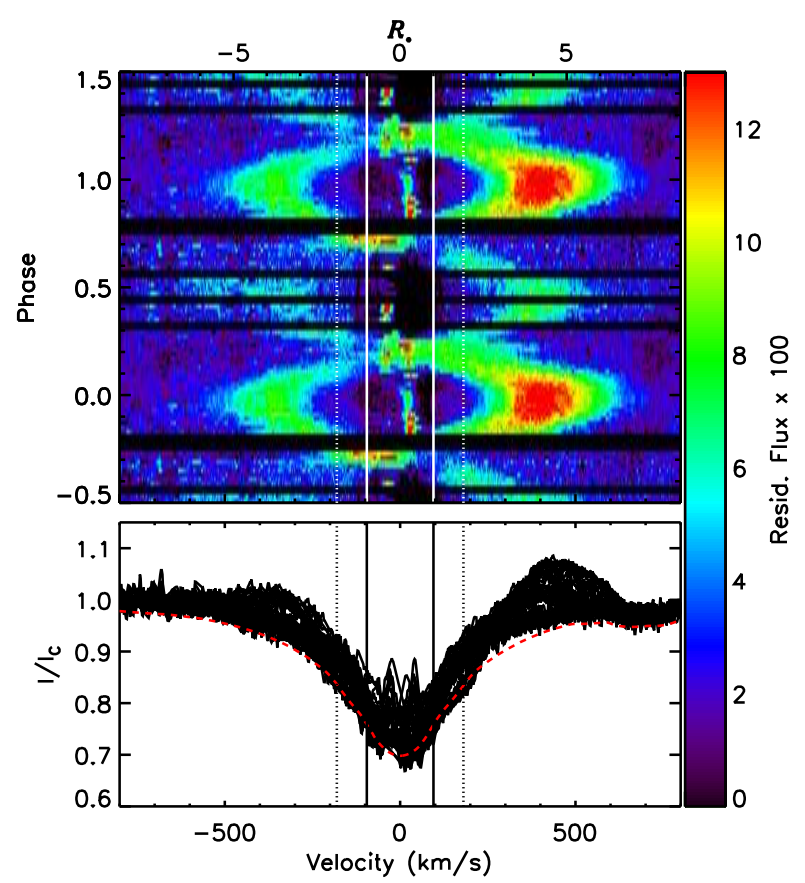

Figure 13. H $\alpha$ dynamic spectrum. The top panel shows residual flux, shifted to the rest frame of $\mathrm{W} 601 \mathrm{~B}$, folded with the rotational period, with intensity corresponding to the colour bar. The bottom panel shows the observed spectra (black) and an example synthetic spectrum (red). Residual flux was determined by subtracting synthetic binary spectra tailored to each observation. The vertical solid and dashed lines show $\pm v \sin i= \pm R_{*}$, and $\pm R_{\mathrm{K}}$, for $\mathrm{W} 601 \mathrm{~B}$. The asymmetry in emission strength between red and blue emission bumps at phase 0 is likely indicative of a departure from a pure dipole. There is additionally no indication of eclipses in the core of the line.

consistent with line formation within a Centrifugal Magnetosphere (CM) formed in a tilted dipole (Petit et al. 2013). In such a case the CM is expected to be shaped like a warped disk, with the two densest regions at the intersections of the rotational and magnetic equators (e.g. Townsend \& Owocki 2005). When the magnetic pole is closest to the line of sight (i.e. at maximum $\left|\left\langle B_{z}\right\rangle\right|$ ), the projected area of the CM, and therefore the $\mathrm{EW}$ of $\mathrm{H} \alpha$, is at a maximum. If the second magnetic pole is visible at some point during the rotation cycle, this should correspond to a secondary emission peak. This pattern is indeed observed in most CM host stars, the exceptions being those stars with very complex surface magnetic fields (Shultz et al. 2020).

From the star's fundamental and atmospheric parameters, the Vink et al. (2001) mass-loss recipe yields a massloss rate of $\log \dot{M}=-9.5 \pm 0.1 \mathrm{M}_{\odot} / \mathrm{yr}$ and a wind terminal velocity of about $1000 \mathrm{~km} \mathrm{~s}^{-1}$. From Eqn. 7 in ud-Doula \& Owocki (2002), the wind magnetic confinement parameter (that is, the ratio of magnetic to kinetic energy density at the magnetic equator at the stellar surface) is $\log \eta_{*}=6.0 \pm 0.2 ;$ since $\eta_{*}$ is greater than unity the wind is magnetically confined. From Eqn. 9 in ud-Doula et al. (2008), $\eta_{*}$ scales to an Alfvén radius (i.e. the maximum extent of magnetic confinement) of $R_{\mathrm{A}}=32 \pm 4 R_{*}$.

The Lorentz force enforces corotation of magnetically confined plasma with the stellar magnetic field out to $R_{\mathrm{A}}$. A CM forms when the Kepler corotation radius $R_{\mathrm{K}}$, de- fined as the distance at which centrifugal and gravitational forces are balanced, is less than $R_{\mathrm{A}}$. The Kepler radius is obtained from the critical rotation parameter $W=$ $v_{\text {orb }} / v_{\text {eq }}=0.24_{-0.01}^{+0.1}$ via the scaling $R_{\mathrm{K}} / R_{*}=W^{-2 / 3}$, where $v_{\text {orb }}=\sqrt{G M_{*} / R_{*}}$ is the orbital velocity i.e. the velocity required for a Keplerian orbit at the stellar equator ud-Doula et al. 2008). The equatorial rotational velocity is $v_{\text {eq }}=161_{-9}^{+32} \mathrm{kms}^{-1}$, with a moderate oblateness ratio of the polar to equatorial radii of $R_{\mathrm{p}} / R_{\mathrm{e}}=0.97 \pm 0.02$. The Kepler corotation radius is then $R_{\mathrm{K}}=2.1 \pm 0.2 R_{*}$ (using Eqn. 14 from ud-Doula et al. 2008). This is a similar Kepler radius to those seen for other $\mathrm{H} \alpha$-bright CM host stars (Shultz et al. 2019d). $R_{\mathrm{K}}$ is indicated in Fig. 9, as expected, the strongest emission is above the Kepler radius. The ratio of $R_{\mathrm{A}}$ to $R_{\mathrm{K}}$ is $\log \left(R_{\mathrm{A}} / R_{\mathrm{K}}\right)=1.2 \pm 0.1$ : not only is $R_{\mathrm{A}}$ significantly greater than $R_{\mathrm{K}}$, but $\log \left(R_{\mathrm{A}} / R_{\mathrm{K}}\right)$ is well within the typical range for $\mathrm{H} \alpha$-bright $\mathrm{CM}$ host stars (Shultz et al. 2019d).

A dynamic spectrum of W $601 \mathrm{~B}$ 's $\mathrm{H} \alpha$ line is shown in Fig. 13. This was created by subtracting the synthetic spectra used to correct the EWs for the RV variation of the two stars ( $\$$ 6. Fig. 9) from the observed spectra, with the residual flux shifted to the rest frame of W601 B. Due to the rigid rotation of the $\mathrm{CM}$, there is a linear relationship between the projected distance from the star and velocity, i.e. $r / R_{*}=v_{\mathrm{r}} / v \sin i$ (where $v_{\mathrm{r}}$ is the line-of-sight velocity), as indicated on the top horizontal axis. At maximum emission, there are two emission bumps extending out to several stellar radii, with the strongest emission at about $3.8 R_{*}$. These emission bumps move closer to the line centre as they weaken in strength, corresponding to the reduction of the projected areas of the clouds simultaneous with the reduction in their projected distance from the star. The secondary emission maximum at phase 0.5 has a similar extent in velocity space, but lower peak emission strength, indicating that the $\mathrm{CM}$ is more flattened in projection at this phase, which is consistent with the negative pole not coming as close to alignment with the line of sight.

There is stronger emission in the rotationally broadened core of the line around phases 0.25 and 0.75 than is present in this part of the line at other phases. There is nebular emission contaminating the very centre of the line (see also Fig. 9), however this nebular emission is much narrower than signatures associated with the CM near phases 0.25 and 0.75 . There are no enhanced absorption features in the line core at these phases, and therefore no evidence that the star is eclipsed by its CM (as seen for $\sigma$ Ori, HR 7355, or HD 176582; Oksala et al. 2012; Rivinius et al. 2013; Bohlender \& Monin 2011). The presence or absence of eclipses is highly dependent on the magnetic geometry. No eclipses are expected if $i_{\text {rot }} \leqslant 35^{\circ}$ (Townsend 2008), as inferred from $\left\langle B_{z}\right\rangle$. On the other hand, the ORM parameters inferred from direct modelling of Stokes $V$ predict two prominent eclipses near phase 0.5 (Townsend 2008).

As noted in $\S 8$, there is some suggestion that W 601 B's surface magnetic field is not purely dipolar. The strong redblue asymmetry in the $\mathrm{H} \alpha$ emission, especially prominent near phase 0.0 (Fig. 13) can only be explained if the surface magnetic field is not a pure dipole.

Shultz et al. (2020) and Owocki et al. (2020) showed that the peak emission strength of stars with CMs is governed by centrifugal breakout, a magnetic reconnection 
W601: a magnetospheric hot PMS binary

mechanism whereby the magnetic field is overloaded by the wind-fed plasma and is explosively ejected away from the star (ud-Doula et al. 2006, 2008). The greater the strength $B_{\mathrm{K}}$ of the magnetic field at the Kepler radius, the greater the density of plasma that the magnetosphere can hold, the more extensive the optically thick part of the $\mathrm{H} \alpha$-emitting $\mathrm{CM}$, and the stronger the emission. W601 B's maximum emission strength is about $0.15 \mathrm{~nm}$. W $601 \mathrm{~B}$ contributes about $33 \%$ of the total light at $\mathrm{H} \alpha$ (as determined from the two stars' radii and synthetic BSTAR2006 spectra for their respective atmospheric parameters), and this emission strength should therefore be scaled up by a factor of about 3 , to a single-star emission strength of $0.45 \mathrm{~nm}$. The strength of the equatorial magnetic field at W601 B's Kepler radius is $\log B_{\mathrm{K}}=2.7 \pm 0.2$, using the dipole strength inferred from $\left\langle B_{z}\right\rangle$. Utilizing the centrifugal breakout emission strength scaling law developed by Owocki et al. (2020), a star with this value of $B_{\mathrm{K}}$ and W $601 \mathrm{~B}$ 's stellar and rotational parameters has an expected emission strength of $0.4 \pm 0.1 \mathrm{~nm}$, almost exactly the measured value. Notably, this emission strength puts W601 B in the company of the $\mathrm{CM}$ host stars with the strongest $\mathrm{H} \alpha$ emission (namely $\sigma$ Ori E and HD 345439; Shultz et al. 2020). Using the dipole strength inferred from Stokes $V$ yields $\log B_{\mathrm{K}}=2.65_{-0.22}^{+0.13}$ and an inferred emission equivalent width of about 0.2 to $0.3 \mathrm{~nm}$, still amongst the strongest known.

The peak emission strength of W 601 B's CM occurs at about $3.8 R_{*}$, which is about $1.8 R_{\mathrm{K}}$. Shultz et al. (2020) noted that $R_{\mathrm{K}}$ is systematically less than the radius of maximum emission, and the discrepancy between the radius of maximum emission and $R_{\mathrm{K}}$ is in this case at the top of the range found for their sample (see their Fig. 12). As discussed above in $\S 8$, the conflict between ORM parameters inferred via modelling of $\left\langle B_{z}\right\rangle$ and via modelling of Stokes $V$ can be reconciled if the radius is smaller than was assumed when deriving ORM parameters from $\left\langle B_{z}\right\rangle$. This smaller radius also yields a larger Kepler radius, $R_{\mathrm{K}}=2.80 \pm 0.03 R_{*}$. This is still only about $70 \%$ of the radius of emission maximum, therefore adopting the minimum possible radius cannot resolve the discrepancy in the case of this star.

Binarity is unlikely to have any effect on W601 B's H $\alpha$ magnetosphere. The periastron separation of the stars is about 0.9 AU (see Tables 2 and 3), whereas the Alfvén radius of $\mathrm{W} 601 \mathrm{~B}$ is a maximum of $37 R_{*}$ or, with $R_{*}=4.4 \mathrm{R}_{\odot}$, about 0.75 AU. W601 A is thus outside of W601 B's magnetosphere at all orbital phases. This argument is of course sensitive to the mass-loss rate prescription: the much lower Krtička (2014) mass-loss rates imply $R_{\mathrm{A}}=62_{-6}^{+22} R_{*}$, i.e. about $1.3_{-0.1}^{+0.4} \mathrm{AU}$, in which case $\mathrm{W} 601 \mathrm{~A}$ is inside the magnetosphere at essentially all orbital phases. In either case, however, W $601 \mathrm{~A}$ is very far from the distance of $\mathrm{H} \alpha$ line formation, a maximum of $7 R_{*}=0.15 \mathrm{AU}$ from $\mathrm{W} 601 \mathrm{~B}$.

It is interesting to note that W601 emits gyrosynchrotron radiation (Kurapati et al. 2017), which is believed to be generated by electrons accelerated to relativistic energies within the middle magnetosphere current sheet just outside the Alfvén radius (Trigilio et al. 2004). If W 601 B's current sheet extends far enough beyond $R_{\mathrm{A}}$ (which is by no means excluded by the models developed by Trigilio et al. 2004), it may be possible that W601 A disrupts the current sheet during periastron passage. It would be of interest to obtain radio observations of this system at apastron and periastron, in order to look for such an effect.

\subsection{Rotational and Magnetic Evolution}

Amongst the magnetic high-mass pre-main sequence stars, W $601 \mathrm{~B}$ has both the strongest magnetic field and one of the shortest rotation periods. Of the magnetic PMS stars noted in the introduction, the mean surface dipole strength is about $1.2 \mathrm{kG}$, and the strongest belongs to V 380 Ori (with a strength of $2.2 \mathrm{kG}$; Alecian et al. 2009), while the measured rotation periods of other magnetic PMS hot stars range from $4 \mathrm{~d}$ to $40 \mathrm{~d}$ (Alecian et al. 2009; Järvinen et al. 2015). This makes W601 B's rotational and magnetic evolution of some interest.

Main sequence magnetic hot stars experience simultaneous decline in surface magnetic field strength, due to a combination of magnetic flux conservation in an expanding atmosphere and magnetic flux decay, and rapid magnetic braking due to angular momentum loss (ud-Doula et al. 2008; Shultz et al. 2019d). As a PMS star, W601 B is still contracting towards the ZAMS. Therefore, flux conservation would imply that its surface magnetic field should grow stronger as the radius shrinks, while at the same time conservation of angular momentum would normally mean that the star should spin up. In this case, the current radius of about $4.4 \mathrm{R}_{\odot}$ should shrink to about $3.2 \mathrm{R}_{\odot}$ at the ZAMS. The total unsigned magnetic flux is $\Phi=B_{\mathrm{d}} R_{*}^{2}$, therefore if flux is conserved then the ZAMS field should increase to about $21_{-2}^{+5} \mathrm{kG}$. This is comparable to the top of the range of surface magnetic dipole strengths of magnetic early B-type stars seen close to the ZAMS (Shultz et al. 2019d).

At the same time, conservation of angular momentum means that the star should spin up as it approaches the ZAMS. Under the assumption that angular momentum is conserved, the ZAMS equatorial rotational velocity should be $v_{\text {eq,ZAMS }}=v_{\text {eq }} R_{*} / R_{\text {ZAMS }}=250_{-15}^{+46} \mathrm{~km} \mathrm{~s}^{-1}$, corresponding to a rotational period $P_{\text {rot,ZAMS }} \sim 0.7 \mathrm{~d}$. This is similar to the two most rapidly rotating magnetic B-type stars known, HR 5907 and HR 7355 Grunhut et al. 2012; Rivinius et al. 2013), which have periods of about $0.5 \mathrm{~d}$.

Angular momentum of course should not be conserved, since it is being lost via the magnetosphere. From Eqn. 20 in ud-Doula et al. (2009), the spindown timescale $\tau_{\mathrm{J}}$ - i.e. the e-folding timescale for the rotation parameter $W$ - is $\log \left(\tau_{\mathrm{J}} / \mathrm{yr}\right)=5.9_{-0.1}^{+0.3}$ or between $700 \mathrm{kyr}$ and $1.5 \mathrm{Myr}$, using the Vink et al. (2001) mass-loss rates. If Krtička (2014) mass-loss rates are used instead, $\log \left(\tau_{\mathrm{J}} / \mathrm{yr}\right)=6.5_{-0.1}^{+0.3}$. Given the current age of the star it should reach the ZAMS in about $300 \mathrm{kyr}$, i.e. about half an e-folding timescale using Vink mass-loss, and much shorter than $\tau_{\mathrm{J}}$ using Krtička massloss. As the star evolves towards the ZAMS, its rotational evolution should therefore be dominated by spin-up due to contraction.

We conducted an analysis of the rotational evolution of W $601 \mathrm{~B}$ using the $7 \mathrm{M}_{\odot}$ PMS Haemmerlé et al. (2019) evolutionary track, simultaneously accounting for angular momentum loss via the magnetosphere, spin-up due to contraction towards the ZAMS, and the change in $B_{\mathrm{d}}$ due to flux conservation. The model had an initial dipole strength $B_{\text {d,init }}=11 \mathrm{kG}$. We started the model at $4 \mathrm{R} \odot$ (corresponding to an age of $400 \mathrm{kyr}$ from the birthline), with $W=0.3$ 
(corresponding to $P_{\text {rot }} \sim 1.13 \mathrm{~d}$ ). The minimum rotation period in this case is achieved at $490 \mathrm{kyr}$, with $P_{\text {rot }} \sim 0.55 \mathrm{~d}$; after this point magnetic braking dominates the rotational evolution. This corresponds to a spin-up of $-0.5 \mathrm{~s} / \mathrm{yr}$. More accurate predictions of the spin-up rate may be provided by the development of a PMS extension of self-consistent stellar evolutionary models incporating fossil fields, similar to those presented by Keszthelyi et al. (2020).

Rotational evolution has been detected in several magnetic early-type stars. $\sigma$ Ori $\mathrm{E}$ is spinning down at a rate of about $+0.08 \mathrm{~s} / \mathrm{yr}$, consistent with expectations from magnetic braking (Townsend et al. 2010). HD 37776 and CU Vir both exhibit complex patterns of cyclical spin-up and spindown, (Mikulášek et al. 2008, 2011, 2017), with typical period changes on the order of $0.6 \mathrm{~s} / \mathrm{yr}$ and $0.1 \mathrm{~s} / \mathrm{yr}$, respectively. HD 142990 is spinning up at a rate of about $-0.6 \mathrm{~s} / \mathrm{yr}$ (Shultz et al. 2019c). It is intriguing to note that both HD 37776 and HD 142990 are very young stars (Shultz et al. 2019d), and their spin-up rates are comparable to that expected for W601 B. While contraction towards the ZAMS cannot explain the cyclical nature of HD 37776's period evolution, it may provide an explanation for that of HD 142990. In all cases in which rotational evolution has been directly measured, the datasets have spanned about 30 years; it is therefore not yet possible to detect rotational evolution in $\mathrm{W} 601 \mathrm{~B}$, since the dataset for this star is extends across only 7 years, with the majority of the data having been acquired over a 2-year time span.

Shultz et al. (2019d) noted that ultra-slow rotators such as $\xi^{1}$ CMa (Shultz et al. 2017, 2018c) are difficult to explain using standard magnetic braking theory. In particular, they are so slowly rotating that their rotation periods are even longer than can be explained by standard magnetic braking theory under the usual assumption of initially critical rotation (ud-Doula et al. 2009; Petit et al. 2013). One possible explanation is that such stars lose a great deal of angular momentum on the PMS. Indeed, their initial critical rotation fraction at the ZAMS would need to already be very close to 0. So far no such magnetic PMS B-type stars have been found (although the magnetic Herbig Ae star HD 101412 is quite a slow rotator, with a period of $42 \mathrm{~d}$; Järvinen et al. 2015). Instead, W $601 \mathrm{~B}$ is apparently a precursor of stars that arrive at the ZAMS as rapid rotators. However, at this point relatively few magnetic PMS hot stars are known; identifying a larger sample is crucial to determining whether there is indeed a sub-population of PMS slow rotators that can serve as the progenitors for stars such as $\xi^{1} \mathrm{CMa}$, or whether some additional braking mechanism is necessary to explain ultra-slow rotation.

\subsection{Implications for the origin of fossil magnetic fields}

Using the same $7 \mathrm{M}_{\odot}$ evolutionary track, once again assuming magnetic flux conservation and evolving back in time from W 601 B's current magnetic parameters, its surface magnetic dipole would have been at a minimum intensity of about $700 \mathrm{G}$ approximately $140 \mathrm{kyr}$ ago. Assuming the same $i_{\text {rot }}$ and $\beta$ as presently, $B_{\mathrm{d}}=700 \mathrm{G}$ would yield a maximum $\left\langle B_{z}\right\rangle$ of about $130 \mathrm{G}$. This is comparable to the typical $\left\langle B_{z}\right\rangle$ values observed by Villebrun et al. (2019) in their study of T-Tauri Stars (TTSs), Intermediate Mass T-Tauri Stars (IMTTSs), and Herbig Ae stars.

This surface dipole strength is also comparable to the mean surface magnetic field strengths of TTSs and IMTTSs, which range from a few hundred $\mathrm{G}$ to a few $\mathrm{kG}$ (Donati et al. 2007, 2008, 2010, 2011b a, 2013, 2015; Hussain et al. 2009; Kochukhov 2015; Hill et al. 2017; Lavail et al. 2017; Yu et al. 2017; Lavail et al. 2020; Johns-Krull et al. 2013). The continuity in total unsigned magnetic flux between stars at various stages on the PMS and MS, and across a wide range of masses, is suggestive of a common origin of fossil magnetic fields in dynamo processes occurring early in the evolutionary process. A striking property of several studies of magnetic binaries is that the magnetic properties of the two stars are often remarkably different even when their masses and rotational properties are nearly identical. For stars with convective envelopes this manifests as one star having a globally organized poloidal field and the other a more tangled magnetic topology. This is so for both V1878 Ori, and the MS M dwarf visual binary GJ65 A and B (Kochukhov \& Lavail 2017). In the case of the B9 V 'identical twin' eclipsing binary HD 62658, one of the two stars is a chemically peculiar Bp star with a strong magnetic field, while the other is a normal, non-magnetic B-type star (Shultz et al. 2019e). Another example is provided by the B2 IV binary HD 149277, with a mass ratio of 1.1, in which one component is a He-strong star with a surface dipole strength of several $\mathrm{kG}$, and the other has no detectable magnetic field (Shultz 2016; González et al. 2018). While the mass ratio of W 601 is much larger than these systems, it follows the general pattern of one component hosting a very strong magnetic field, while the upper limit for the magnetic field of the other is comparable to the $300 \mathrm{G}$ critical field strength and therefore probably on the order of a few $G$ (§8.1), and confirms that this pattern is established already on the PMS.

The existence of several close binaries containing a magnetic star poses a challenge to the scenario in which dynamos generated during binary mergers are the primary pathway to fossil magnetic fields (e.g. Schneider et al. 2019), since mergers should not produce close binaries. Shultz et al. (2019e) suggested that the properties of the binaries above point to an alternative scenario. During the convective stage of the star's evolution, irrespective of rotational properties or stellar mass, the dynamo magnetic field spontaneously settles into one of two attractor states: a globally organized dipole, or a tangled topology. Both leave behind a fossil field when the star becomes fully radiative. However, weaker or more tangled fields rapidly decay under the influence of rotational or convective instabilities (e.g. Aurière et al. 2007; MacDonald \& Petit 2019; Jermyn \& Cantiello 2020). In the case of close binaries, flux decay is accelerated via tidally induced instabilities (Vidal et al. 2019). The result is that, very shortly after the cessation of convective support for a dynamo, only the strongest and most organized magnetic fields survive, while the incidence of magnetic fields amongst close binary stars is decreased even further by the additional influence of tidal instabilities. The abrupt change in magnetic incidence from essentially $100 \%$ to the canonical $10 \%$ seen for MS hot stars is precisely what is seen at the boundary between stars with convective envelopes and fully radiative stars (Villebrun et al. 2019). 


\subsection{Formation of surface chemical abundance peculiarities}

The existence of He spots on the surface of W601 B, as inferred from the rotational modulation of the He I 667.8 nm line (Fig. 11), indicates that surface chemical abundances become established quite rapidly on the PMS following the formation of the radiative envelope. It is instructive in this regard to compare $\mathrm{W} 601 \mathrm{~B}$ with other magnetic hot stars on the PMS. HD 72106 A (B9p) is the magnetic primary of a PMS SB2 system, with a non-magnetic Herbig Ae companion (Folsom et al. 2008). The primary, which is either just reaching or has just passed the ZAMS, already possesses strong chemical peculiarities, while the non-magnetic companion has normal chemical abundances. This is similar to the case of W 601. By contrast, the magnetic Herbig Ae star HD 190073 has normal chemical abundances (Catala et al. 2007; Folsom et al. 2012), while another magnetic Herbig Ae star, HD 101412 (Wade et al. 2005; Hubrig et al. 2009), is underabundant in some elements (Cowley et al. 2010; Folsom et al. 2012). The abundances of HD 101412 appear to reflect those of its dustdepleted accreting material (Kama et al. 2015).

Notably, both HD 190073 and HD 101412 possess emission lines, which are most likely formed in accretion disks. HD 72106 A does not show Herbig emission, and while $\mathrm{W} 601 \mathrm{~B}$ is an $\mathrm{H} \alpha$-bright star its emission originates in a $\mathrm{CM}$ and not an accretion disk. The scenario that emerges from this comparison is one in which magnetic hot stars that are still accreting possess surface abundances reflecting the accreting material. Once accretion ceases, diffusion in the magnetically stabilized radiative envelope leads to the rapid emergence of surface chemical peculiarities. Another interesting point of comparison is the PMS Am star Stock 1612 (Netopil et al. 2014). While Am stars do not possess strong magnetic fields, the existence of chemical peculiarities on a non-accreting PMS star is further evidence that these can form rapidly once accretion has ceased.

A possible contradiction to this scenario is V 380 Ori A, which shows both weak Bp-type chemical peculiarities (Folsom et al. 2012) and very strong Herbig-type $\mathrm{H} \alpha$ emission. However, the secondary of this system is a chemically normal A-type star, which is much closer to the birthline, and it is very possible that the Herbig emission originates from this component.

Atomic diffusion in PMS stars was evaluated by Vick et al. 2011) in order to determine how quickly the Ap/Bp phenomenon can be established. They found that, so long as turbulence is suppressed, surface chemical abundance peculiarities can form within 20 to $2 \mathrm{Myr}$, with the timescale decreasing with increasing stellar mass. While the minimum timescale is significantly longer than the PMS lifetime of W $601 \mathrm{~B}$, the models utilized by Vick et al. (2011) extended only up to $2.8 \mathrm{M}_{\odot}$, much lower than W601 B's mass. Although models of higher-mass PMS stars are needed to explore the parameter space occupied by W601 B, the trend of decreasing timescales with increasing mass suggests that the formation of chemical spots on W601 B's surface within a few hundred kyr is probably not in conflict with our current understanding. It is worth noting that suppression of turbulence within subsurface opacity-bump convection zones is both expected (MacDonald \& Petit 2019;
Jermyn \& Cantiello 2020) and observed (Sundqvist et al. 2013) in strongly magnetic early-type stars, and that the criterion that turbulence be suppressed is therefore very likely to be fulfilled.

\section{CONCLUSIONS}

We have analyzed a large spectropolarimetric dataset of the magnetic pre-Main Sequence (PMS) star W 601. The system turns out to be a spectroscopic binary with a mass-ratio of 1.8 and an orbital period of about $110 \mathrm{~d}$. Atmospheric parameters $\left(T_{\text {eff }}\right.$ and $\log g$ ) were determined for the two stars via spectroscopic analysis. The $T_{\text {eff }}$ of the primary, W $601 \mathrm{~A}$, is about $22 \mathrm{kK}$, consistent with previous determinations for this star; the secondary, W601 B, is slightly cooler at about $19 \mathrm{kK}$. W $601 \mathrm{~A}$ has a mass of about $12 \mathrm{M}_{\odot}$, and W $601 \mathrm{~B}$ a mass of about $7 \mathrm{M}_{\odot}$.

Magnetic analysis reveals that the magnetic field belongs to $\mathrm{W} 601 \mathrm{~B}$. The peak $\left\langle B_{z}\right\rangle$ measured from disentangled LSD profiles is about $2 \mathrm{kG}$.

W 601 was originally classified as a Herbig Be star by Alecian et al. (2008b) due to its $\mathrm{H} \alpha$ emission and midinfrared excess. However, close examination of $\mathrm{H} \alpha$ shows that it varies coherently with $\left\langle B_{z}\right\rangle$, and displays the typical features of a centrifugal magnetosphere. W 601 is therefore not a classical Herbig Be star. However, both components have low surface gravities, around 3.8. Such a low $\log g$ indicates either that the two stars are very evolved (around 22 Myr old) or still contracting towards the main sequence. Only the latter hypothesis is consistent with W 601's membership in the NGC 6611 open cluster, which has a mainsequence turnoff age of 2 to 4 Myr reflecting the oldest populations in the cluster, but also contains ongoing star formation. Even if W 601 is not a member of the cluster, if the stars are on the MS the low $\log g$ implies that W601 A must be near the TAMS; given the mass ratio, W $601 \mathrm{~B}$ should still be near the ZAMS and have a much higher surface gravity than is consistent with the data. Spectroscopic modelling furthermore indicates that the two stars have similar radii, which again is only possible if they are still on the PMS. Therefore, while W 601 is not a classical Herbig Be star, the orbital and spectoscopic properties of the system indicate that it is probably a PMS star as suggested by Martavan et al. (2008) and Alecian et al. (2008b).

A rotational period of about $1.13 \mathrm{~d}$ was determined from $\mathrm{H} \alpha$ and $\left\langle B_{z}\right\rangle$. Modelling of $\left\langle B_{z}\right\rangle$ using this period and W 601 B's stellar parameters yields $B_{\mathrm{d}} \sim 11 \mathrm{kG}$. This makes W $601 \mathrm{~B}$ by far the most strongly magnetic of the known magnetic PMS stars. When the magnetic star's emission strength is corrected for dilution by the non-magnetic primary, W $601 \mathrm{~B}$ is also revealed to have $\mathrm{H} \alpha$ emission comparable to the strongest seen in the population of CM host stars. This extremely strong emission is consistent with the star's rapid rotation, strong surface magnetic field, mass, and radius. Indeed, the star's emission strength is very close to the value predicted both by empirical (Shultz et al. 2020) and theoretical (Owocki et al. 2020) analyses of similar stars based on the centrifugal breakout mass balancing mechanism.

While $\left\langle B_{z}\right\rangle$ is consistent with a tilted dipole, Stokes $V$ is not satisfactorily reproduced by a purely dipolar model. 
Strong red-blue asymmetry in the $\mathrm{H} \alpha$ emission is furthermore indicative that the surface magnetic field is not purely dipolar. This indicates that distortions to the magnetic dipole are already present on the PMS.

$\mathrm{W} 601 \mathrm{~B}$ is a He-strong star with weakly variable $\mathrm{He}$ lines, indicating that surface chemical abundance peculiarities are also already established on the PMS. We were unable to detect indications of significant departure from solar abundances in other spectroscopic lines, or statistically significant variability in the stronger metallic lines. This may indicate that the star is still in the process of forming its surface chemical abundance spots, and that so far only the He spots have become noticeable. On the other hand, the low $S / N$ of the data may simply make metallic abundance patches difficult to detect.

Extrapolation of W601 B's magnetic and rotational properties as it contracts towards the ZAMS indicate that it should arrive on the MS with a surface dipole magnetic field strength of about $20 \mathrm{kG}$ and a rotational period of about 0.6 d. Thus, when $\mathrm{W} 601 \mathrm{~B}$ arrives on the MS, it will have amongst the most extreme magnetic and rotational properties of any known magnetic hot star. W $601 \mathrm{~B}$ is therefore a precursor to objects such as $\sigma$ Ori E, HR 5907, or HR 7355. The expected rate of rotational spin-up, about $-0.5 \mathrm{~s} / \mathrm{yr}$, is similar to the rate recently determined by Shultz et al. (2019c) for HD 142990 and is in principle detectable if the star is monitored over the next ten or twenty years.

\section{ACKNOWLEDGEMENTS}

The authors acknowledge the numerous helpful comments made by the referee, Dr. Gregory Herczeg, which have greatly improved the quality of this paper. This work is based on observations obtained at the Canada-FranceHawaii Telescope (CFHT) which is operated by the National Research Council of Canada, the Institut National des Sciences de l'Univers (INSU) of the Centre National de la Recherche Scientifique (CNRS) of France, and the University of Hawaii. The MiMeS collaboration acknowledges financial support from the Programme National de Physique Stellaire (PNPS) of INSU/CNRS. MES acknowledges financial support from the Annie Jump Cannon Fellowship, supported by the University of Delaware and endowed by the Mount Cuba Astronomical Observatory. EA acknowledges financial support from 'Programme National de Physique Stellaire' (PNPS) of CNRS/INSU (France). VP acknowledges support from the National Science Foundation under Grant No. 1747658. GAW acknowledges support from the Natural Sciences and Engineering Research Council (NSERC) of Canada in the form of a Discovery Grant.

\section{DATA AVAILABILITY STATEMENT}

Reduced ESPaDOnS spectra are available at the CFHT archive maintained by the CADC at https://www.cadc-ccda.hia-iha.nrc-cnrc.gc.ca/en/, where they can be found via standard stellar designations.

\section{REFERENCES}

Alecian E., et al., 2008a, MNRAS 385, 391

Alecian E., et al., 2008b, A\&A 481, L99

Alecian E., et al., 2009, MNRAS 400, 354

Alecian E., et al., 2013a, MNRAS 429, 1001

Alecian E., Wade G. A., Catala C., Grunhut J. H., Landstreet J. D., Böhm T., Folsom C. P., Marsden S., 2013b, MNRAS, 429, 1027

Aurière M., et al., 2007, A\&A 475, 1053

Bagnulo S., Landstreet J. D., Fossati L., Kochukhov O., 2012, A\&A, 538, A129

Blazère A., Neiner C., Petit P., 2016, MNRAS, 459, L81

Blazère A., Petit P., Neiner C., Folsom C., Kochukhov O., Mathis S., Deal M., Landstreet J., 2020, MNRAS 492, 5794

Bloomfield P., 1976, Fourier analysis of time series: an introduction. Wiley Series in Probability and Mathematical Statistics, New York: Wiley

Bohlender D. A., Monin D., 2011, AJ, 141, 169

Bonnell I. A., Bate M. R., 1994, MNRAS, 271, 999

Bouvier J., et al., 2007, A\&A, 463, 1017

Braithwaite J., Spruit H. C., 2004, Nature, 431, 819

Breger M., et al., 1993, A\&A, 271, 482

Cantat-Gaudin T., et al., 2018, A\&A, 618, A93

Catala C., et al., 2007, A\&A, 462, 293

Cowley C. R., Hubrig S., González J. F., Savanov I., 2010, A\&A, 523, A65

Cutri R. M., et al., 2003, VizieR Online Data Catalog, 2246, 0

Donati J.-F., Semel M., Rees D. E., 1992, A\&A, 265, 669

Donati J.-F., Semel M., Carter B. D., Rees D. E., Collier Cameron A., 1997, MNRAS, 291, 658

Donati J. F., et al., 2007, MNRAS, 380, 1297

Donati J. F., et al., 2008, MNRAS 386, 1234

Donati J. F., et al., 2010, MNRAS 409, 1347

Donati J. F., et al., 2011a, MNRAS 417, 472

Donati J. F., et al., 2011b, MNRAS 417, 1747

Donati J. F., et al., 2013, MNRAS 436, 881

Donati J. F., et al., 2015, MNRAS, 453, 3706

Dufton P. L., et al., 2006, A\&A 457, 265

Ekström S., et al., 2012, A\&A 537, A146

Evans C. J., et al., 2005, A\&A, 437, 467

Fitzpatrick E. L., Massa D., 1999, ApJ, 525, 1011

Folsom C. P., Wade G. A., Kochukhov O., Alecian E., Catala C., Bagnulo S., Landstreet J. D., Hanes D., 2008, Contributions of the Astronomical Observatory Skalnate Pleso, 38, 245

Folsom C. P., Bagnulo S., Wade G. A., Alecian E., Landstreet J. D., Marsden S. C., Waite I. A., 2012, MNRAS, 422, 2072

Folsom C. P., Likuski K., Wade G. A., Kochukhov O., Alecian E., Shulyak D., 2013, MNRAS, 431, 1513

Fossati L., et al., 2015, A\&A 574, A20

Gaia Collaboration et al., 2018, A\&A, 616, A1

Getman K. V., Feigelson E. D., Kuhn M. A., Bate M. R., Broos P. S., Garmire G. P., 2018, MNRAS, 476, 1213

González J. F., Levato H., 2006, A\&A, 448, 283

González J. F., Hubrig S., Järvinen S. P., Schöller M., 2018, MNRAS, 481, L30

Grunhut J. H., et al., 2012, MNRAS, 419, 1610

Grunhut J. H., et al., 2017, MNRAS, 465, 2432 
W601: a magnetospheric hot PMS binary

Guarcello M. G., Prisinzano L., Micela G., Damiani F., Peres G., Sciortino S., 2007, A\&A, 462, 245

Haemmerlé L., et al., 2019, A\&A 624, A137

Hill C. A., Carmona A., Donati J. F., Hussain G. A. J., Gregory S. G., Alencar S. H. P., Bouvier J., Matysse Collaboration 2017, MNRAS, 472, 1716

Hillenbrand L. A., Massey P., Strom S. E., Merrill K. M., 1993, AJ, 106, 1906

Høg E., et al., 2000, A\&A, 355, L27

Horne J. H., Baliunas S. L., 1986, ApJ, 302, 757

Hubrig S., et al., 2009, A\&A 502, 283

Hubrig S., Ilyin I., Schöller M., Lo Curto G., 2013, Astronomische Nachrichten, 334, 1093

Hubrig S., Carroll T. A., Scholler M., Ilyin I., 2015, MNRAS, 449, L118

Hussain G. A. J., et al., 2009, MNRAS 398, 189

Järvinen S. P., Carroll T. A., Hubrig S., Schöller M., Ilyin I., Korhonen H., Pogodin M., Drake N. A., 2015, A\&A 584, A15

Jermyn A. S., Cantiello M., 2020, ApJ, 900, 113

Johns-Krull C. M., et al., 2013, ApJ 765, 11

Kama M., Folsom C. P., Pinilla P., 2015, A\&A, 582, L10

Keszthelyi Z., et al., 2020, MNRAS 493, 518

Kochukhov O., 2015, A\&A, 580, A39

Kochukhov O., Lavail A., 2017, $A p J L, 835$, L4

Kochukhov O., Makaganiuk V., Piskunov N., 2010, A\&A 524, A5

Kochukhov O., et al., 2015, A\&A 574, A79

Kochukhov O., Shultz M., Neiner C., 2019, A\&A 621, A47

Krtička J., 2014, A\&A, 564, A70

Kuhn M. A., Hillenbrand L. A., Sills A., Feigelson E. D., Getman K. V., 2019, ApJ, 870, 32

Kumar B., Sagar R., Sanwal B. B., Bessell M. S., 2004, MNRAS, 353, 991

Kupka F. G., Piskunov N., Ryabchikova T. A., Stempels H. C., Weiss W. W., 1999, A\&AS 138, 119

Kupka F. G., Ryabchikova T. A., Piskunov N. E., Stempels

H. C., Weiss W. W., 2000, Balt. Astron., 9, 590

Kurapati S., et al., 2017, MNRAS 465, 2160

Kuschnig R., Weiss W. W., Gruber R., Bely P. Y., Jenkner H., 1997, A\&A, 328, 544

Landstreet J. D., Borra E. F., 1978, ApJL, 224, L5

Landstreet J. D., Bagnulo S., Andretta V., Fossati L., Mason E., Silaj J., Wade G. A., 2007, A\&A, 470, 685

Landstreet J. D., et al., 2008, A\&A, 481, 465

Lanz T., Hubeny I., 2007, ApJS, 169, 83

Lavail A., Kochukhov O., Hussain G. A. J., Alecian E., Herczeg G. J., Johns-Krull C., 2017, A\&A, 608, A77

Lavail A., Kochukhov O., Hussain G. A. J., Argiroffi C., Alecian E., Morin J., BinaMIcS Collaboration 2020, MNRAS, 497, 632

Lenz P., Breger 2005, Communications in Asteroseismology, 146, 53

Lignières F., Petit P., Böhm T., Aurière M., 2009, A\&A, 500, L41

MacDonald J., Petit V., 2019, MNRAS 487, 3904

Maíz Apellániz J., Barbá R. H., 2018, A\&A, 613, A9

Martayan C., Floquet M., Hubert A. M., Neiner C., Frémat

Y., Baade D., Fabregat J., 2008, A\&A, 489, 459

Martin A. J., et al., 2018, MNRAS 475, 1521

Mathys G., 1989, FCPh, 13, 143
Mendigutía I., Eiroa C., Montesinos B., Mora A., Oudmaijer R. D., Merín B., Meeus G., 2011a, A\&A, 529, A34

Mendigutía I., Calvet N., Montesinos B., Mora A., Muzerolle J., Eiroa C., Oudmaijer R. D., Merín B., 2011b, A\&A, 535, A99

Mestel L., 1999, Stellar magnetism. Oxford : Clarendon, 1999. (International series of monographs on physics ; 99)

Mikulášek Z., et al., 2008, A\&A, 485, 585

Mikulášek Z., et al., 2011, A\&A, 534, L5

Mikulášek Z. Z., Krtička J., Janík J., Henry G. W., Zejda M., Shultz M., Paunzen E., Jagelka M., 2017, in Balega Y. Y., Kudryavtsev D. O., Romanyuk I. I., Yakunin I. A., eds, Astronomical Society of the Pacific Conference Series Vol. 510, Stars: From Collapse to Collapse. p. 220 (arXiv:1702.02195)

Nazé Y., Petit V., Rinbrand M., Cohen D., Owocki S., udDoula A., Wade G. A., 2014, ApJS 215, 10

Neiner C., Mathis S., Alecian E., Emeriau C., Grunhut J., BinaMIcS MiMeS Collaborations 2015, in Nagendra K. N., Bagnulo S., Centeno R., Jesús Martínez González M., eds, IAU Symposium Vol. 305, Polarimetry. pp 61-66 (arXiv:1502.00226), doi:10.1017/S1743921315004524

Neiner C., et al., 2017, MNRAS 471, 1926

Netopil M., Fossati L., Paunzen E., Zwintz K., Pintado O. I., Bagnulo S., 2014, MNRAS 442, 3761

Nieva M.-F., 2013, A\&A, 550, A26

Oksala M. E., Wade G. A., Townsend R. H. D., Owocki S. P., Kochukhov O., Neiner C., Alecian E., Grunhut J., 2012, MNRAS, 419, 959

Owocki S. P., Shultz M. E., ud-Doula A., Sundqvist J. O., Townsend R. H. D., Cranmer S. R., 2020, MNRAS 499, 5366

Pablo H., et al., 2019, MNRAS 488, 64

Pecaut M. J., Mamajek E. E., 2013, ApJS, 208, 9

Petit V., Wade G. A., 2012, MNRAS, 420, 773

Petit V., Wade G. A., Drissen L., Montmerle T., Alecian E., 2008, MNRAS, 387, L23

Petit P., et al., 2010, A\&A, 523, A41

Petit P., et al., 2011, A\&A, 532, L13

Petit V., et al., 2013, MNRAS 429, 398

Petit V., et al., 2019, MNRAS 489, 5669

Piskunov N. E., Kupka F., Ryabchikova T. A., Weiss W. W., Jeffery C. S., 1995, A\&AS, 112, 525

Preston G. W., 1967, ApJ, 150, 547

Preston G. W., 1974, ARAA, 12, 257

Reeve D. C., Howarth I. D., 2016, MNRAS, 456, 1294

Rivinius T., Townsend R. H. D., Kochukhov O., Stefl S., Baade D., Barrera L., Szeifert T., 2013, MNRAS, 429, 177

Rusomarov N., Kochukhov O., Ryabchikova T., Piskunov N., 2015, A\&A 573, A123

Rusomarov N., Kochukhov O., Ryabchikova T., Ilyin I., 2016, A\&A, 588, A138

Rusomarov N., Kochukhov O., Lundin A., 2018, A\&A, 609, A88

Ryabchikova T. A., Piskunov N. E., Kupka F., Weiss W. W., 1997, Balt. Astron., 6, 244

Ryabchikova T., Piskunov N., Kurucz R. L., Stempels H. C., Heiter U., Pakhomov Y., Barklem P. S., 2015, Phys. Scr., 90, 054005

Schneider F. R. N., Ohlmann S. T., Podsiadlowski P., Röpke F. K., Balbus S. A., Pakmor R., Springel V., 2019, 
Nature, 574, 211

Schöller M., et al., 2016, A\&A, 592, A50

Shultz M., 2016, PhD thesis, Queen's University (Canada

Shultz M., et al., 2015a, MNRAS, 449, 3945

Shultz M., Wade G. A., Alecian E., BinaMIcS Collaboration 2015b, MNRAS 454, L1

Shultz M., Wade G. A., Rivinius T., Neiner C., Henrichs H., Marcolino W., MiMeS Collaboration 2017, MNRAS 471, 2286

Shultz M., Rivinius T., Wade G. A., Alecian E., Petit V., 2018a, MNRAS, 475, 839

Shultz M. E., et al., 2018b, MNRAS, 475, 5144

Shultz M., Kochukhov O., Wade G. A., Rivinius T., 2018c, MNRAS, 478, L39

Shultz M., et al., 2019a, MNRAS, 482, 3950

Shultz M. E., et al., 2019b, MNRAS, 485, 1508

Shultz M., Rivinius T., Das B., Wade G. A., Chand ra P., 2019c, MNRAS, 486, 5558

Shultz M. E., et al., 2019d, MNRAS, 490, 274

Shultz M. E., et al., 2019e, MNRAS 490, 4154

Shultz M. E., et al., 2020, MNRAS 499, 5379

Sikora J., Wade G. A., Power J., Neiner C., 2019a, MNRAS, 483, 2300

Sikora J., Wade G. A., Power J., Neiner C., 2019b, MNRAS, 483, 3127

Silvester J., Kochukhov O., Rusomarov N., Wade G. A., 2017, MNRAS, 471, 962

Stibbs D. W. N., 1950, MNRAS, 110, 395

Sundqvist J. O., Petit V., Owocki S. P., Wade G. A., Puls J., MiMeS Collaboration 2013, MNRAS, 433, 2497

Townsend R. H. D., 2008, MNRAS, 389, 559

Townsend R. H. D., Owocki S. P., 2005, MNRAS, 357, 251

Townsend R. H. D., Oksala M. E., Cohen D. H., Owocki S. P., ud-Doula A., 2010, ApJL, 714, L318

Trigilio C., Leto P., Umana G., Leone F., Buemi C. S., 2004, A\&A, 418, 593

Vick M., Michaud G., Richer J., Richard O., 2011, A\&A 526, A37

Vidal J., Cébron D., ud-Doula A., Alecian E., 2019, A\&A 629, A142

Villebrun F., et al., 2019, A\&A, 622, A72

Vink J. S., de Koter A., Lamers H. J. G. L. M., 2001, A\&A, 369,574

Vink J. S., Drew J. E., Harries T. J., Oudmaijer R. D., Unruh Y., 2005, MNRAS, 359, 1049

Wade G. A., Donati J.-F., Landstreet J. D., Shorlin S. L. S., 2000, MNRAS, 313, 851

Wade G. A., et al., 2005, A\&A 442, L31

Wade G. A., et al., 2016, MNRAS 456, 2

Wichittanakom C., Oudmaijer R. D., Fairlamb J. R., Mendigutía I., Vioque M., Ababakr K. M., 2020, MNRAS, 493, 234

Wolff S. C., Strom S. E., Dror D., Venn K., 2007, AJ 133, 1092

Yu L., et al., 2017, MNRAS 467, 1342

de Winter D., Koulis C., The P. S., van den Ancker M. E., Perez M. R., Bibo E. A., 1997, A\&AS, 121, 223

ud-Doula A., Owocki S. P., 2002, ApJ, 576, 413

ud-Doula A., Townsend R. H. D., Owocki S. P., 2006, ApJl 640, L191

ud-Doula A., Owocki S. P., Townsend R. H. D., 2008, MNRAS, 385, 97
ud-Doula A., Owocki S. P., Townsend R. H. D., 2009, MNRAS, 392, 1022 